\title{
Atmospheres of Brown Dwarfs
}

\author{
Christiane Helling · Sarah Casewell
}

[Received:] Version: 26 September 2014 / Accepted: 22 October 2014

\begin{abstract}
Brown Dwarfs are the coolest class of stellar objects known to date. Our present perception is that Brown Dwarfs follow the principles of star formation, and that Brown Dwarfs share many characteristics with planets. Being the darkest and lowest mass stars known makes Brown Dwarfs also the coolest stars known. This has profound implication for their spectral fingerprints. Brown Dwarfs cover a range of effective temperatures which cause brown dwarfs atmospheres to be a sequence that gradually changes from a M-dwarf-like spectrum into a planet-like spectrum. This further implies that below an effective temperature of $\lesssim 2800 \mathrm{~K}$, clouds form already in atmospheres of objects marking the boundary between M-Dwarfs and brown dwarfs. Recent developments have sparked the interest in plasma processes in such very cool atmospheres: sporadic and quiescent radio emission has been observed in combination with decaying Xray-activity indicators across the fully convective boundary.
\end{abstract}

Keywords (Stars:) brown dwarfs - Stars: low-mass - Stars: atmospheres - infrared: stars $\cdot$ Radio lines: stars $\cdot$ X-rays: stars

ChH highlights financial support of the European Community under the FP7 by an ERC starting grant. SLC acknowledges financial support of University of Leicester. V. Wild is thanked for 'large-scale metallicity gradients'. M. Marley, A. Scholz, I. Vorgul, P. Rimmer, T. Robinson, I. Leonhardt are thanked for reading the manuscript. The authors thank D. Saumon, M. Cushing and J.D. Kirkpatrick for kindly providing spectra. This research has benefited from the SpeX Prism Spectral Libraries, maintained by Adam Burgasser and the IRTF spectral library maintained by Mike Cushing. Most literature search was performed using the NASA Astrophysics Data System ADS. Our local computer support is highly acknowledged.

Ch. Helling

SUPA, University of St Andrews, North Haugh, St Andrews, KY16 9SS, UK

Tel.: +123-45-678910

Fax: +123-45-678910

E-mail: ch80@st-and.ac.uk

S. Casewell

Department of Physics and Astronomy, University of Leicester, University road, Leicester, LE1 7RH 


\section{Introduction}

Brown dwarfs are free-floating stellar objects with masses below the hydrogen-burning limit of $\sim 0.075 \mathrm{M}_{\odot}$ and with radii of $\approx 1 R_{\text {Jup }}$ for mature objects, although this can vary with cloud cover and metallicity. They are the low-mass extension of the subsolar main-sequence in the Herzsprung-Russel diagram (HRD, Fig. 11. Their total emitted flux, and hence their effective temperature $\mathrm{T}_{\text {eff }}$, is lower than that of $\mathrm{M}$ dwarfs. Brown dwarfs become increasingly dimmer as they age because their mass is too small to sustain continuous hydrogen burning. Only in their youth, the heaviest brown dwarfs fuse some helium and maybe lithium. Hence, the class of brown dwarfs comprises members that are just a little cooler than M-dwarfs (L dwarfs) and members that can be as cold as planets ( $\mathrm{T}$ and $\mathrm{Y}$ dwarfs). Several formation mechanisms are suggested, including the classical star-forming scenario of a local gravitational collapse of an interstellar molecular cloud. The formation efficiency may have changed depending on time and location. The oldest brown dwarfs could be as old as the first generation of stars that formed in the universe. Their metallicity would be extremely low leaving the spectrum almost feature-less (Fig. 9 in Witte et al 2009). Luhman (2012) reviews the formation and evolution of brown dwarfs, including the initial mass function and circumstellar disks. Observational evidence builds up for that brown dwarfs and giant gas planets overlap in masses and in global temperatures (see review Chabrier et al 2014). Joergens (2014) reviews the emergence of brown dwarfs as a research area started by a theoretical prediction of their existence, and emphasizes the research progress in formation and evolution of brown dwarfs. Allard et al (1997) reviewed model atmospheres of very low mass stars and brown dwarfs discussing model aspects like updating gas-phase opacities, convection

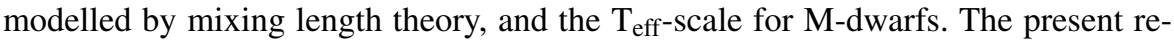
view summarizes the progress in brown dwarf observations extending now from the UV into the radio, revealing new atmospheric processes and indicating overlapping parameter ranges between brown dwarfs and planets (Sect. 2). Special emphasis is given to cloud modelling as part of the brown dwarf atmospheres, a field with increasing importance since the first review on brown dwarf atmospheres by Allard et al (1997) (Sect. 3). Since Allard et al (1997), a considerably larger number of brown dwarfs is known which allows first statistical evaluations, one example being the search for correlation between X-ray emission and rotational activity in brown dwarfs. Wavelength-dependent variability studies have gained momentum, and the idea of weather on brown dwarfs is commonly accepted since the first variability search by Tinney and Tolley (1999a). In the following, we summarize the observational achievements for field brown dwarfs. We ignore the specifics of young brown dwarfs as this is reviewed in e.g. Luhman (2012) and concentrate on evolved brown dwarfs, not on individual star forming regions or brown dwarfs with disks. Sections 4 discusses the idea of model benchmarking as it emerged in the brown dwarf community. Section 5 gives an outlook regarding new challenges like multi-dimensional atmosphere modelling and kinetic gas-phase chemistry. 


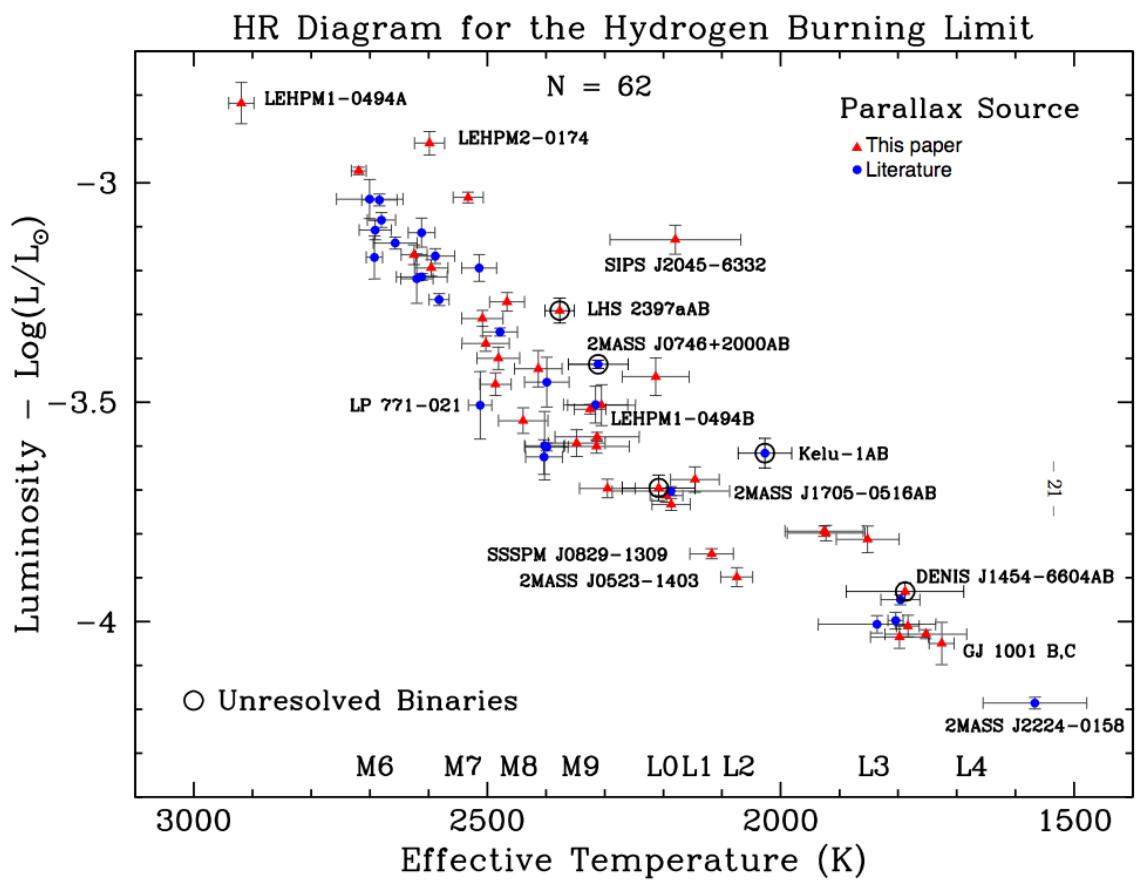

Fig. 1 The low-mass end of the main sequence in the HRD diagram showing the transition from the stellar M-dwarf to the substellar brown dwarf regime (Dieterich et al 2013). 


\section{Brown dwarf observations in different spectral energy ranges}

In this section of the review we aim to bring the reader up to date with the observations of brown dwarfs. We describe the recent results focusing on cloud effects in low gravity objects, and their comparison with young extrasolar planets, as well as observations of low metallicity objects. We also discuss the recent reports of photometric and spectroscopic variability of brown dwarfs, which is linked to patchy clouds, temperature fluctuations within the atmosphere and weather effects, as well as high energy phenomena that are linked to emissions seen in the radio, X-ray and UV wavelength regimes.

\subsection{Optical and IR spectral types}

Although the first brown dwarf to be discovered was the L4 dwarf GD165B (Becklin and Zuckerman, 1988), it was not identified as such until the discovery of Gl229B (Nakajima et al, 1995) and Teide 1 (Rebolo et al, 1995) in 1995. Since then, astronomers have searched for a way of classifying brown dwarfs. These objects are very different from the $\mathrm{M}$ dwarfs known at the time, and the contrast between the dusty $\mathrm{L}$ dwarfs and the methane rich $\mathrm{T}$ dwarfs is stark. Both these spectral types are discussed extensively in the literature and are not described here in great detail (Burrows et al 2001: Lodders and Fegley 2006 for an overview).

The $\mathrm{L}$ dwarfs are similar to $\mathrm{M}$ dwarfs in photospheric chemical composition, containing alkali lines of $(\mathrm{K}, \mathrm{Na})$ and metal hydrides $(\mathrm{FeH})$ and oxides (TiO, $\mathrm{VO})$ and water. As we progress through the spectral types from $\mathrm{L} 0$ to $\mathrm{L} 9$, the $\mathrm{TiO}$ and VO bands weaken, the alkali lines become weaker and more pressure broadened, and the water bands and $\mathrm{FeH}$ strengthen in the optical. In the near-IR, $\mathrm{CO}$ strengthens towards the mid-L dwarfs, and then weakens again as methane begins to form. The change between the $\mathrm{L}$ and $\mathrm{T}$ dwarfs, often called the L-T transition region is characterized by the near-infrared colours of the brown dwarfs changing, while the effective temperature of the objects remains the same (Figure2, see Kirkpatrick et al 1999 for a review). T dwarfs, sometimes called methane dwarfs are characterized by the methane absorption seen in the near-IR that gets progressively stronger as one progresses through the subclasses, making the $J-H$ and $H-K$ colours bluer. In the optical, the spectrum is affected by collisionally induced molecular hydrogen absorption and $\mathrm{FeH}$.

2011, marked the discovery of an additional, and later spectral type, the $\mathrm{Y}$ dwarf. There are $\sim 20 \mathrm{Y}$ dwarfs known to date (Cushing et al, 2011; Luhman et al, 2011; Kirkpatrick et al, 2012, 2013; Cushing et al, 2014). The majority of these have spectral types ranging between $\mathrm{Y} 0$ and $\mathrm{Y} 2$ and were discovered using the Wide-field Infrared Survey Explorer (WISE). WISE was designed to discover Y dwarfs: its shortest wavelength band at $3.4 \mu \mathrm{m}$ was selected to fall in the centre of the fundamental methane absorption band at $3.3 \mu \mathrm{m}$ and the W2 filter at $4.6 \mu \mathrm{m}$ detects radiation from the deeper, hotter layers in the atmosphere. When combined, the W1-W2 colour is very large, allowing the detection of Y dwarfs (Kirkpatrick et al, 2011). 
All the $\mathrm{Y}$ dwarfs show deep $\mathrm{H}_{2} \mathrm{O}$ and $\mathrm{CH}_{4}$ absorption bands in their near-infrared spectra, similar to late-T dwarfs (Figure 2). These water clouds were measured in detail by Morley et al (2014b). The $J$ band peaks of the Y dwarfs are narrower than those of the latest type T dwarfs, and the ratio of the $J$ and $H$ band flux is close to 1 , meaning that the $J-H$ trend towards the blue for T dwarfs turns back towards the red for Y0. This trend also occurs for the $Y-J$ colour. This colour reversal is thought to be caused by alkali atoms, that normally dominate the absorption in the shorter wavelengths, being bound in molecules, thus reducing the alkali atom opacity (Liu et al, 2010). While the $H$ band spectra of T dwarfs are shaped by $\mathrm{CH}_{4}$ and $\mathrm{H}_{2} \mathrm{O}$, for $\mathrm{Y}$ dwarfs as the effective temperature decreases, $\mathrm{NH}_{3}$ absorption becomes important (Lodders and Fegley, 2002; Burrows et al, 2003).

Cushing et al (2011) estimate $\mathrm{T}_{\text {eff }}=350 \ldots 500 \mathrm{~K}$ for the $\mathrm{Y} 0 \mathrm{dwarfs}$, with their masses between $\sim 5$ and $20 \mathrm{M}_{\text {Jup }}$. However, using a luminosity measurement derived from a Spitzer based parallax, Dupuy and Kraus (2013) estimate their effective temperatures to be typically $60-90 \mathrm{~K}$ hotter. This difference in temperature is possibly caused by using near-IR spectra, a regime where only $5 \%$ of the Y dwarf flux is emitted and so models do not always accurately reproduce the observations (Dupuy and Kraus, 2013). Using models containing sulphide and chloride clouds from Morley et al (2012), Beichman et al (2014) obtain the lower effective temperatures, suggesting that previous results apply on a model dependent bolometric correction. There is one other Y dwarf, not discovered by WISE: WD0806-661B, which was until recently, the most likely candidate for the lowest mass and temperature Y dwarf at 6-10 $\mathrm{M}_{\mathrm{Jup}}$ and 330-375 K, although there are as yet no spectra of this object. This has since been superseded by the discovery of WISEJ085510.83-071442.5, a high proper motion Y dwarf at $2 \mathrm{pc}$. This object is our fourth nearest neighbour and has an estimated effective temperature of 225-260 K and a mass of 3-10 $\mathrm{M}_{\text {Jup }}$ (Luhman, 2014). 


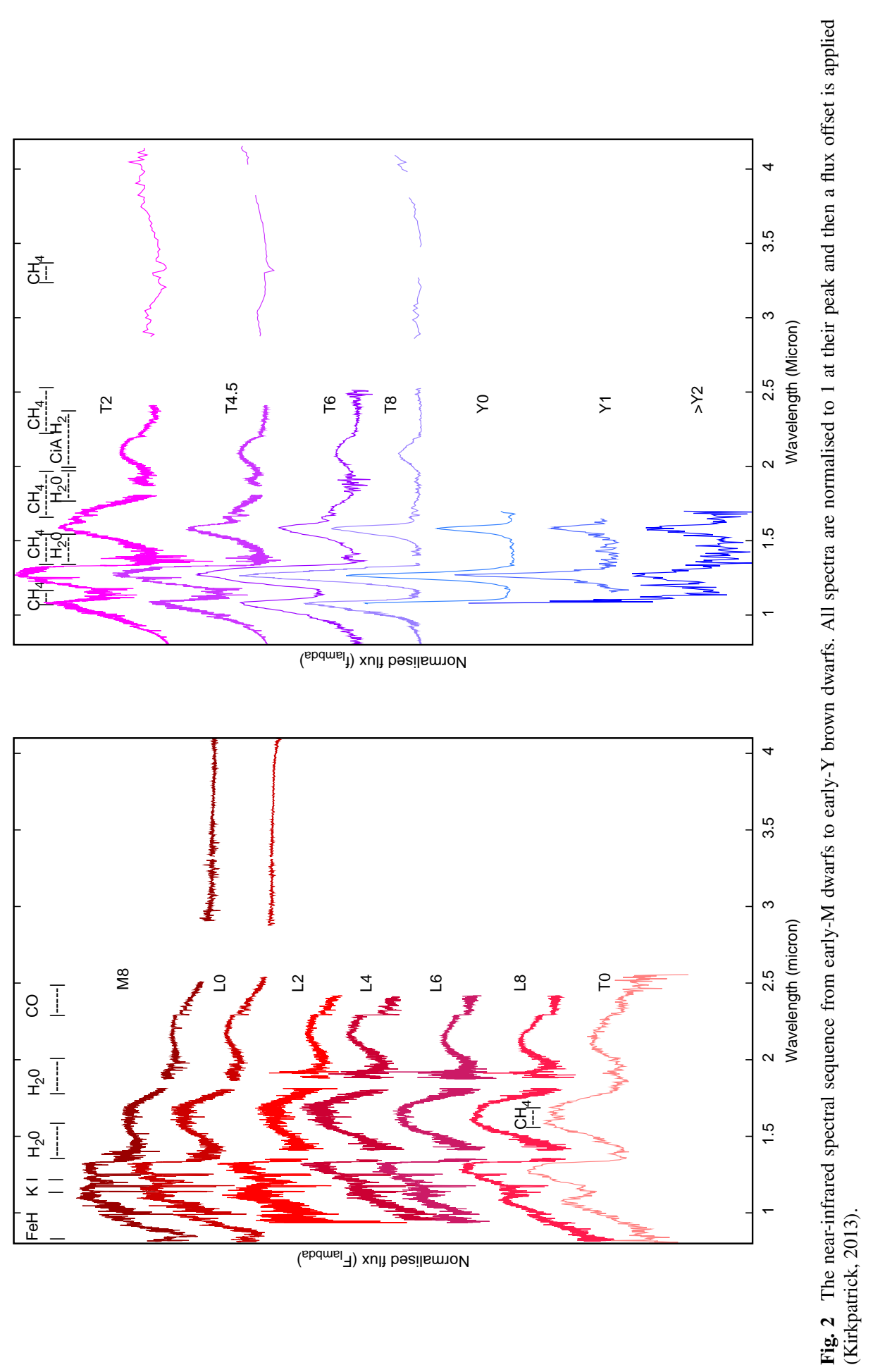




\subsubsection{Brown dwarf classification}

Brown dwarf spectral types do not fit into the standard Morgan-Keenan stellar spectral types system because their age-mass-temperature degeneracy causes the classical stellar mass-temperature relationship to break down for such ultra-cool objects. An object of a specific spectral type (or effective temperature) may be higher mass and old, or lower mass and young. For instance, an L5 dwarf $\left(\mathrm{T}_{\mathrm{eff}} \sim 1500 \mathrm{~K}\right)$ in the Pleiades cluster (125 Myr) has a mass of $25 \mathrm{M}_{\mathrm{Jup}}$, but a field dwarf of the same spectral type is much more massive at $70 \mathrm{M}_{\text {Jup }}$ (Chabrier et al, 2000).

There are in general three methods of identifying a brown dwarf's spectral type: the first, by comparison with spectral templates as is usual for stars that fit the MorganKeenan classification scheme, the second is by using indices derived from spectral parameters and the third is by comparing broadband photometry to spectral standards. The first two methods are described for the L dwarf classification in the optical wavelength range by Kirkpatrick et al (1999) and Martín et al (1999), respectively. The most commonly used method is to compare spectra of objects to "standard" or "template" spectra and to use spectral indices as a secondary calibration tool, for instance to judge metallicity or gravity (see Sect.2.1.3 for the gravity classification scheme). The template scheme was extended into the near-IR by Reid et al (2001), and the indices by Geballe et al (2002). This index scheme is now more widely used than that of Martín et al (1999) and extends down to T9. Burgasser et al (2006) combined the index scheme of Geballe et al (2002) with their template (Burgasser et al, 2002) to create a unified way of spectral typing $\mathrm{T}$ dwarfs. When using these methods to classify $\mathrm{L}$ and $\mathrm{T}$ dwarfs it should be noted that the assigned spectral types are limited to only the parts of the spectrum that are measured. For objects on the L-T transition, it is not unusual to have optical and near-IR derived spectral types that differ by $>1$ spectral type.

\subsubsection{Low metallicity brown dwarfs}

Low metallicity brown dwarfs (subdwarfs) provide an insight into the coolest, oldest brown dwarfs. The existence of low-metallicity brown dwarfs indicates that such low-mass stars also formed in a younger universe when the metallicity was lower than today. It is further of interest to comparing their atmospheres to those of low metallicity extrasolar planets which formed as by-product of star formation.

Only a handful of ultracool subdwarfs are known to date (Burgasser et al, 2003, Burgasser, 2004, Cushing et al, 2009, Sivarani et al, 2009; Lodieu et al, 2010; Kirkpatrick et al, 2010, Lodieu et al, 2012; Mace et al, 2013; Kirkpatrick et al, 2014, Burningham et al, 2014). Of the $\sim 30$ objects known to date, only 11 have spectral types later than L2 (Kirkpatrick et al, 2014, Burningham et al, 2014; Mace et al, 2013). The naming scheme for subdwarfs follows that for $M$ dwarfs developed by Gizis (1997) and upgraded by (Lépine et al, 2007), moving from dM for metal rich M dwarfs to subdwarfs (sdM), extreme subdwarfs (esdM) and ultra subdwarfs (usdM) in order of decreasing metallicity.

Burgasser et al (2008) noted that L subdwarfs are overluminous in $M_{J}$, but slightly underluminous in $M_{K}$. This change is suggested to be caused by a reduced cloud opac- 


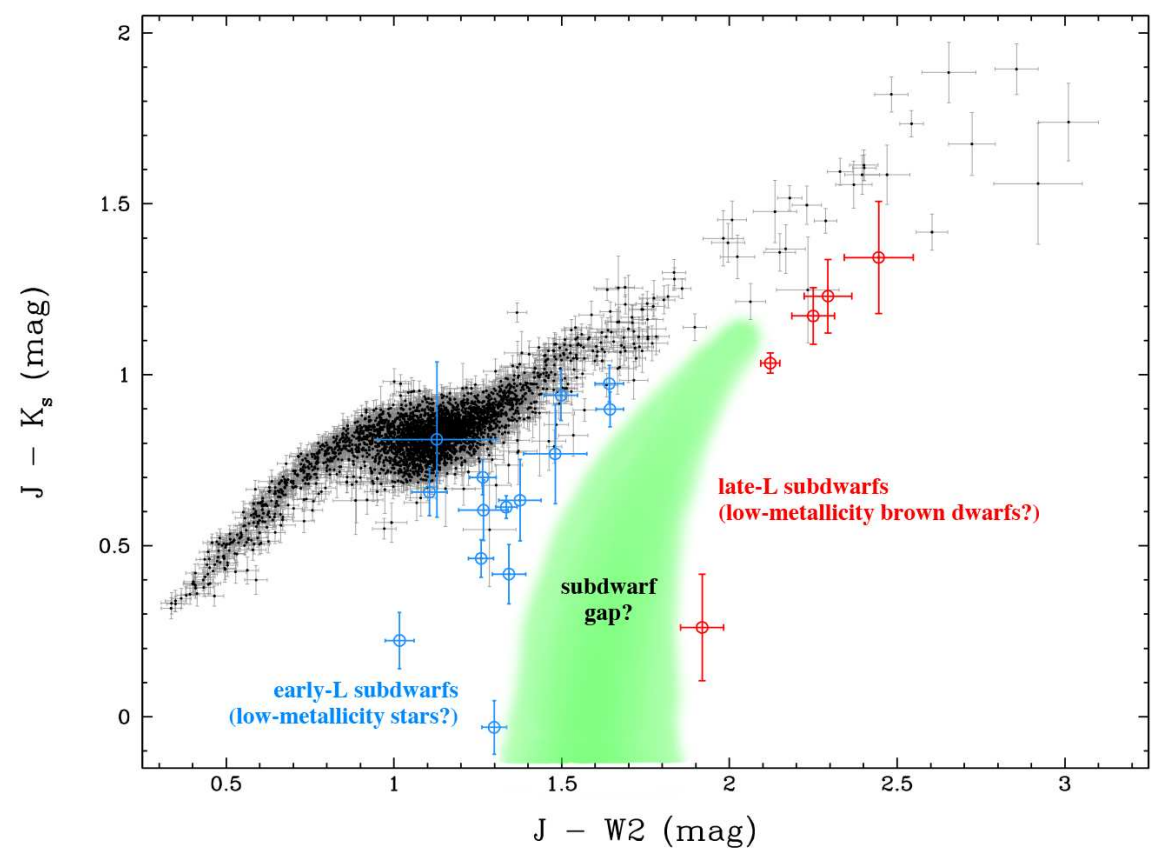

Fig. 3 The effective temperature gap between brown dwarfs and M-dwarfs increases with lower metallicity (lower J-K $\mathrm{K}_{\mathrm{s}}$ ). Known late-M (SpecT $<$ sdL5 - blue circles) and L subdwarfs (SpecT $>$ sdL5 - red circles) and AllWISE proper motion stars (solid black dots) are shown (Kirkpatrick et al 2014). Kirkpatrick et al (2014) suggest that the wedge (green zone) covers an area in the diagram where L subdwarfs may rarely be found.

ity causing strong $\mathrm{TiO}, \mathrm{FeH}, \mathrm{Ca}$ I and Ti I features, and enhanced collisional-induced $\mathrm{H}_{2}$ opacity in the $K$ band as predicted by Ackerman and Marley (2001); Tsuji et al (1996b). These sources of opacity will be discussed in more detail in Section 3 Kirkpatrick et al (2014) suggests that an L subdwarf "gap" exists between the early L and late L subdwarfs. This could be explained if lower metallicity brown dwarfs would generally be the older objects. For older brown dwarfs, an effective temperature gap is observed to occur between the hotter, deuterium-burning subdwarfs (blue symbols in Fig. 3), and the coolest, lowest mass members of similarly aged hydrogen burning stars (M-dwarfs, red symbols in Fig. 3). This temperature gap is predicted to increase for older populations Kirkpatrick et al (2014).

Recently, a population of T subdwarfs has emerged (Burningham et al, 2014. Mace et al, 2013). T dwarf colours are very sensitive to small changes in metallicity - a shift of 0.3 dex can change the $H-$ [4.5] colours of T8 dwarfs as much as a $100 \mathrm{~K}$ change in effective temperature (Burningham et al, 2013). The $\mathrm{T}$ dwarfs exhibit enhanced $Y$ and depressed $K$ band flux, indicative of a high gravity, and hence older age, and low metallicity atmosphere (Burgasser et al, 2002). The increased $Y$ band flux is caused as the lower metallicity reduces the opacity in the wings of the alkali lines, resulting in a brighter and broader peak flux (Burgasser and Kirkpatrick, 2006). The 
$K$ band flux depression is created as pressure-enhanced collision induced absorption by molecular hydrogen becomes more important, as molecular features are removed from the spectra (Saumon et al, 1994).

\subsubsection{The surface gravity of brown dwarfs}

Young, low gravity brown dwarfs have very similar properties to directly-imaged exoplanets (Faherty et al, 2013b) and it has been suggested that younger brown dwarfs $(\log (g) \approx 3)$ may have thicker clouds in their atmospheres than those present in older objects $(\log (g) \approx 5)$ of the same effective temperature (Barman et al, 2011, Currie et al, 2011; Madhusudhan et al, 2011). Helling et al (2011b) demonstrate that the geometric cloud extension ${ }^{1}$ increases with decreasing surface gravity $\log (g)$ in cloud-forming atmospheres, an effect likened to an increasing pressure scale height $\mathrm{H}_{\mathrm{P}} \sim 1 / g$, and Marley et al (2012) suggest that these clouds persist for longer, at higher temperatures, than in older objects.

Many brown dwarfs have been identified in young open star clusters (e.g. Sigma Ori: (Bihain et al, 2009, Peña Ramírez et al, 2012), Serpens: (Spezzi et al, 2012); Pleiades: (Casewell et al, 2011,2007); see Luhman et al 2012 for a review). However, there also exists a field population of low gravity, young brown dwarfs (e.g. Reid et al 2008; Cruz et al 2009, Kirkpatrick et al 2010). A comprehensive scheme for defining the gravity of these objects was devised by Kirkpatrick et al (2008) and Cruz et al (2009) in the optical, and Allers and Liu (2013a b) in the near-infrared. The classification introduces a suffix of $\alpha, \beta$, or $\gamma$ to the spectral type, indicating the gravity. $\alpha$ implies a normal gravity field dwarf, whereas $\beta$ is an intermediate-gravity object and $\gamma$ represents a low-gravity object. These suffixes can also be used as a proxy for age.

In the optical, the suffixes are assigned based on measurements of the $\mathrm{Na}$ I doublet and the K I doublet which are weaker and sharper in a low gravity object, the $\mathrm{VO}$ absorption bands which are stronger, and the $\mathrm{FeH}$ absorption features which are weaker. We note that decreasing hydride molecules are typical for low metallicity stars of higher mass, hence higher temperature. The abundance of such hydrogenbinding molecules decreases in brown dwarfs because cloud formation decreases the metal components available.

In the near-infrared, the $\mathrm{VO}$ and $\mathrm{FeH}$ bands are considered simultaneously with the alkali lines to derive $\log (\mathrm{g})$. The changing shape of the $H$-band is also taken into consideration. It becomes more triangular-shaped caused by increasing water absorption as a sign of low gravity (Lucas et al, 2001, Rice et al, 2011). All of these features are altered as there is less pressure broadening due to the object having a low surface gravity causing the pressure scale height to increase (Rice et al, 2011, Allers and Liu, 2013a). In general, alkali absorption features are weaker, and the overall colours of lower gravity objects are redder than those of their higher gravity counterparts (Faherty et al, 2013c). The redder colour is due, in part, to the changes in

\footnotetext{
1 The geometrical cloud extension, or cloud height, can be defined in various ways. Woitke and Helling (2004) used the degree of condensation for Ti (their Eq. 16) for defining the cloud height. The distance between the gas pressure at the nucleation maximum and the gas pressure where all cloud particles have evaporated determine the cloud height in Helling et al (2011b.
} 
the near-infrared broadband features, but also due to more photospheric dust (Helling et al, 2011b). An additional feature is that while young $\mathrm{M}$ dwarfs $(\log (\mathrm{g}) \approx 3)$ are brighter than their older $(\log (g) \approx 4.5)$ counterparts, young L dwarfs $(\log (g) \approx 3)$ are underluminous in the near-infrared for their spectral type. This may be due to the additional dust in their photosphere (Faherty et al, 2013a) or due to a cooler spectral type/temperature relation being required.

There are a handful of directly-imaged planetary mass companions that have estimated effective temperatures in the brown dwarf regime (e.g. Bonnefoy et al 2013), for example 2M1207b ( $10 \mathrm{Myr}, \sim 1600 \mathrm{~K}$, SpecT $\sim$ mid L) and HR8799b $(\sim 30$ Myr, $\sim 1600 \mathrm{~K}$, SpecT $\sim$ early T). These planets are underluminous and have unusually red near-infrared colours, when compared to field brown dwarfs, as well as displaying the characteristic peaked $H$-band spectra. There are $\sim 30$ brown dwarfs that have been kinematically linked to moving groups and association aged between 10 and $150 \mathrm{Myr}$, that share these features, thus indicating that low gravity brown dwarfs may provide a clue to the atmospheric processes occurring on young exoplanets.

\subsection{High energy processes in non-accreting brown dwarfs}

Despite brown dwarfs being objects that are brightest in the near-IR, this has not prevented searches for other types of emission, particularly those associated with higher energy processes such as those seen in early $M$ dwarfs. However, the magnetic dynamo mechanism is used to explain magnetic field generation in solar-type stars, and there is a direct correlation between rotation and magnetic activity indicated by $\mathrm{H} \alpha$, X-rays and radio emission (Noyes et al, 1984, Stewart et al, 1988; James et al, 2000, Delfosse et al, 1998; Pizzolato et al, 2003; Browning et al, 2010; Reiners et al, 2009; Morin et al, 2010). This relationship between the radio $\left(\mathrm{L}_{v, R}\right)$ and X-ray $\left(\mathrm{L}_{X}\right)$ luminosities holds for active F-M stars, and $\mathrm{L}_{X} \propto L_{v, R}^{\alpha}(\alpha \sim 0.73)$ is known as the GüdelBenz relation (Figure 4 Güdel and Benz 1993; Benz and Güdel 1994). As the dynamo operates at the transition layer between the radiative and convective zones (the tachocline), this mechanism cannot explain radio activity in fully convective dwarfs (>M3), and although $\mathrm{H} \alpha$ and $\mathrm{X}$-ray activity continues into the late $\mathrm{M}$ dwarf regime, the tight correlation between $\mathrm{X}$-ray and radio luminosity breaks down which suggests that a separate mechanism is likely to be responsible for radio emission.

\subsubsection{X-ray and UV observations}

Many searches for X-ray emission in L dwarfs were conducted (Stelzer and Neuhäuser, 2003; Berger et al, 2005; Stelzer et al, 2006), but only one detection is known to date. The L dwarf binary Kelu-1, composed of two old brown dwarfs, was detected with Chandra in the energy range of $0.63,0.86,1.19$ and $1.38 \mathrm{keV}$, resulting in an estimation of the $0.1-10 \mathrm{keV}$ X-ray luminosity to be $L_{X}=2.9 \times 10^{25} \mathrm{erg} \mathrm{s}^{-1}$ (Audard et al, 2007). It has been suggested that this emission does not originate from flares as there was no concurrent radio detection at a frequency $\sim 8 \mathrm{GHz}$ (Audard et al, 2007). Audard et al (2007) suggested that the ratio between luminosity (radio, $\mathrm{H} \alpha$, or Xrays) and bolometric luminosity $\left(\mathrm{L} / \mathrm{L}_{\mathrm{bol}}\right)$ increases with decreasing effective temper- 


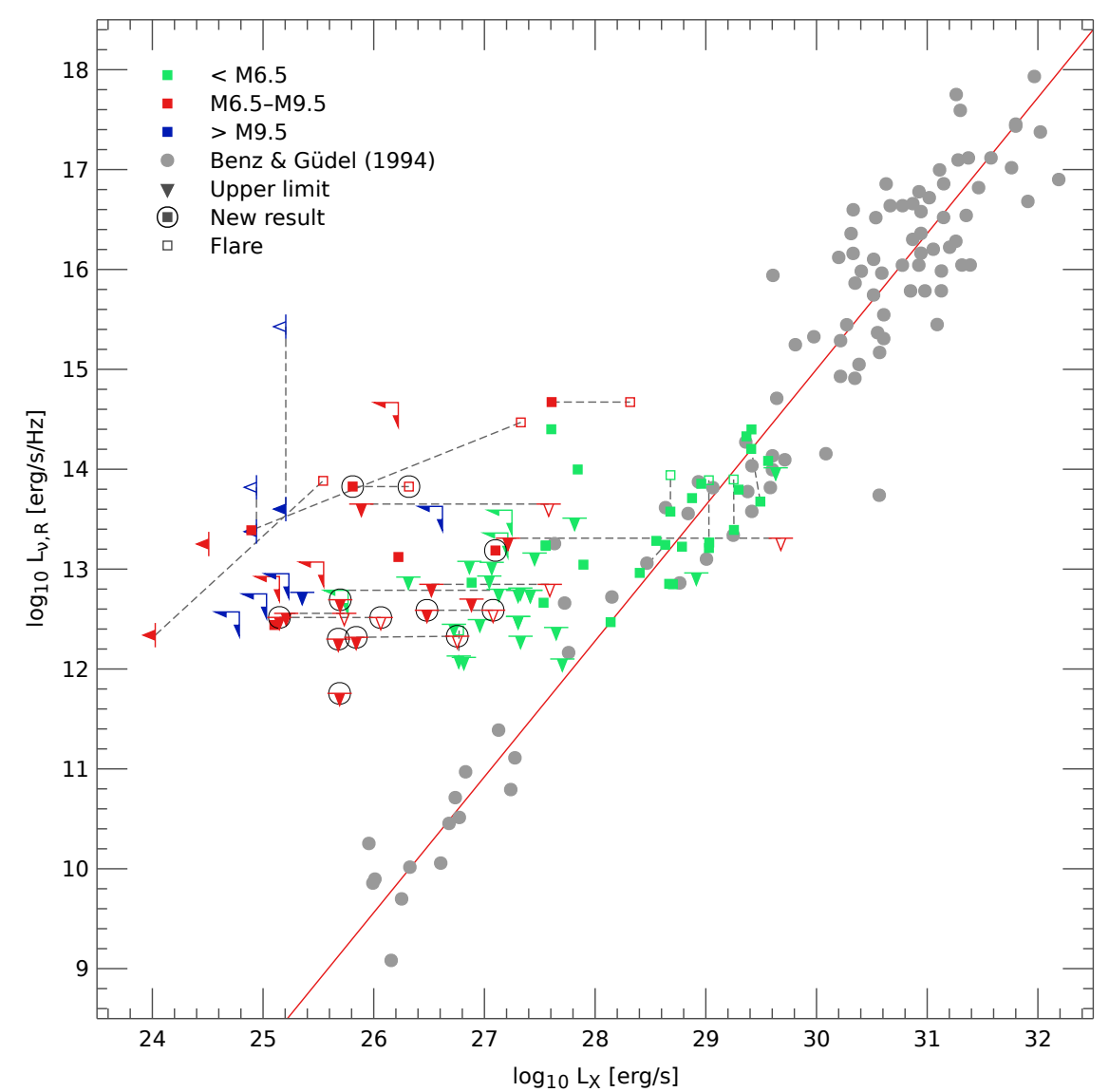

Fig. 4 The Güdel-Benz relationship between $L_{X}(0.2-2 \mathrm{keV})$ and $L_{v, R}$ Williams et al 2013b). Limits are shown as downward pointing triangles. Objects with spectral types of M6 or earlier (green), M6.5-M9.5 dwarfs (red) and spectral types of L0 or later (blue) are shown. Grey circles show the original Güdel-Benz relation from Benz and Güdel (1994). Dashed lines connect multiple measurements of the same source.

ature. They concluded that the chromospheric magnetic activity ( $\mathrm{H} \alpha$ emission) and the activity in the hot coronal loops (X-ray emission) decreases with effective temperature, indicating a different mechanism responsible for the radio emission in ultra cool dwarfs.

Williams et al (2013b) suggest that ultracool dwarfs with strong axisymmetric magnetic fields tend to have $L_{v, \text { Radio }} / L_{X}$ consistent with the Güdel-Benz relation (Fig. 4), while dwarfs with weak non-axisymmetric fields are radio luminous. Slower rotating dwarfs have strong convective field dynamos and so also stay near the Güdel-Benz relation, whereas some rapid rotators may violate the Güdel-Benz relation. Williams et al (2013b) also note that dwarfs with a weaker magnetic field tend to have later spectral types, and lower X-ray luminosity, which may be related 
to their cooler temperatures. They also note that in general, radio-bright sources, tend to be X-ray under luminous compared to radio dim brown dwarf sources.

There are as yet no UV detections of brown dwarfs where the emission is attributed to atmospheric processes. Only brown dwarfs with disks such as the young TW Hydra member 2MASS1207334-39 have been observed to show UV emission. $\mathrm{H}_{2}$ fluorescence is detected and attributed to accretion (Gizis et al, 2005).

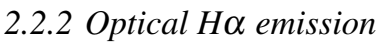

The $\mathrm{H} \alpha$ luminosity (indicative of chromospheric activity) in late $\mathrm{M}$ and $\mathrm{L}$ dwarfs also decreases with lower mass and later spectral type. Schmidt et al (2007) estimate that of their 152 strong sample, $95 \%$ of $\mathrm{M} 7$ dwarfs show $\mathrm{H} \alpha$ emission (consistent with Gizis et al 2000). This fraction decreases with spectral type to 8\% of L2-L8 dwarfs showing $\mathrm{H} \alpha$. For the $\mathrm{L}$ dwarfs in particular, $50 \%$ of $\mathrm{L} 0$ dwarfs being active declines to $20-40 \%$ of L1 dwarfs and only $10 \%$ of L2 dwarfs and later spectral types. This decline is similar to the breakdown in the rotation-activity relationship seen for the $\mathrm{X}$-ray activity (Section 2.2.1) and has been attributed to the high electrical resistivities in the cool, neutral atmospheres of these dwarfs (Mohanty et al, 2002).

Sorahana et al (2014) have recently suggested that molecules may be affected by chromospheric activity. The active chromosphere heats the upper atmosphere, causing the chemistry in that region to change, resulting in the weakening of the $2.7 \mu \mathrm{m}$ water, $3.3 \mu \mathrm{m}$ methane and $4.6 \mu \mathrm{m} \mathrm{CO}$ absorption bands as seen in AKARI spectra of mid-L dwarfs.

Despite the predicted decrease in $\mathrm{H} \alpha$ emission, some objects of late spectral type show $\mathrm{H} \alpha$ emission. For example, the L5 dwarfs 2MASSJ01443536-07 and 2MASS1315-26. In quiescence, these objects show $\mathrm{H} \alpha$ fluxes similar to those of other dwarfs of the same spectral type. However in outburst, 2MASS0144-07 has an $\mathrm{H} \alpha$ flux measurement more than 10 times higher than the mean (Liebert et al, 2003), and for 2MASS1315-26 the $\mathrm{H} \alpha$ emission is $\sim 100$ times stronger than for L dwarfs of a similar spectral type (Hall, 2002).

\subsubsection{Radio emission}

A number of brown dwarfs have been detected to be radio loud. This non-thermal emission may be low level quiescent (Berger, 2002), exhibiting variability (Antonova et al, 2007), showing variability that is periodic and linked to rotation (Berger et al, 2005; McLean et al, 2011), highly polarised and bursting (Burgasser and Putman, 2005), pulsing synchronised with the rotation period, and highly polarised (Hallinan et al, 2007; Berger et al, 2009) or a combination of some of these (Williams et al, 2013a). Some of these objects (TVLM513-46546, 2MASSJ0036+18, LSRJ1835+3259, 2MASSJ1047539+21 Route and Wolszczan 2012, Hallinan et al 2008) emit periodic, $100 \%$ polarised radiation, normally detected at $4-8 \mathrm{GHz}$ with spectral luminosities of $\approx 10^{13.5} \mathrm{erg} \mathrm{s}^{-1} \mathrm{~Hz}^{-1}$ (Hallinan et al, 2006, Route and Wolszczan, 2012). These pulses are caused by the cyclotron maser instability (CMI), the emission mechanism that occurs on Jupiter (Treumann, 2006; Nichols et al, 2012; Morioka et al, 2012). CMI emission requires a relatively tenuous population of energetic particles 
confined to a relatively strong magnetic field; in particular, the cyclotron frequency, $f_{\mathrm{ce}}=e B / m_{e} c$, must be much greater than the plasma frequency $f_{\mathrm{pe}}=\sqrt{4 \pi e^{2} n_{e} / m_{e}}$. Whenever detailed observations are available, the free energy in the plasma is seen to be provided by electrons moving along the magnetic field lines, which can originate in magnetospheric-ionospheric (M-I) shearing and possibly plasma instabilities. These observations suggest that BDs can self-generate stable, $\sim \mathrm{kG}$-strength magnetic fields (Berger, 2006). The underlying assumption is, however, that enough free charges are present to form a plasma in these extremely cold atmospheres $(<2000$ $\mathrm{K})$.

Although this mechanism for emission is quite well characterised, and can account for the polarised flaring behaviour, two of these dwarfs also produce quiescent, moderately polarised emission. This indicates that a second mechanism such as synchrotron or gyrosynchrotron emission (Berger, 2002; Osten et al, 2006; Ravi et al, 2011) may be occurring, or that the CMI emission is becoming depolarised in some way as it crosses the dwarf's magnetosphere (Hallinan et al, 2008). It has been suggested that some of the variability seen in these sources may be due to variation in the local plasma conditions (Stark et al, 2013), perhaps linked to magnetic reconnection events (Schmidt et al, 2007) or due to other sporadic charged events in the plasma (Helling et al 2011b a, 2013; Bailey et al 2013).

It is still unknown what features distinguish radio "active" from radio "inactive" dwarfs. The relationship may depend on mass, effective temperature, activity, magnetic field strength and rotation rate. All the known radio "active" dwarfs have a high vsini-value, indicating short rotation periods ( $\sim 3 \mathrm{hrs}$ ) (Antonova et al, 2013 . Williams, 2013). This may indicate a link between rotation rate and emission, but could also indicate a dependence on the inclination angle, $i$, instead of the velocity, thus making detection of radio emission dependent on the line of sight and the beamed radiation emitted (Hallinan et al, 2008).

\subsection{Observed variability in Brown Dwarfs}

The number of campaigns that search for spectro-photometric variability has increased since it was first suggested by Tinney and Tolley (1999b). Such variability would indicate non-uniform cloud cover (e.g. Ackerman and Marley 2001) and weatherlike features, such as those seen on Jupiter. The majority of the early searches concentrated on L dwarfs, suggesting that variability could be due to holes in the clouds (Gelino et al, 2002; Ackerman and Marley, 2001). Surveys suggest that between 40$70 \%$ of L dwarfs are variable (Bailer-Jones and Mundt, 2001, Gelino et al, 2002, Clarke et al, 2002), although most of these surveys involve small numbers of objects and the authors vary on what is considered a detection. The majority of these studies were performed in the $I$ band where the amplitude of variability is at the 1-2\% level (Clarke et al, 2002, 2008) on timescales of tens of minutes to weeks, and is in general not periodic. There has now been a shift towards using the near-IR for variability studies (Enoch et al, 2003; Clarke et al, 2008; Khandrika et al, 2013; Girardin et al, 2013, Buenzli et al, 2014, Radigan et al, 2014, Wilson et al, 2014) where the frequency of variability is estimated to be $\sim 10-40 \%$. So far, however, high amplitude 
periodic variability $(>3 \%)$ has been limited to the L-T transition objects, while lower amplitude variability is detected in both early $\mathrm{L}$ and late $\mathrm{T}$ dwarfs.

Heinze et al (2013) reported sinusoidal variability of the L3 dwarf DENIS-P $\mathrm{J} 1058.7-1548$ in the $J$ and [3.6] micron bands, but no variability in the [4.5] micron band. They suggested that the variability may again be due to inhomogenous cloud cover, where the thickest clouds have the lowest effective temperature, but there may also be an effect related to magnetic activity (starspots, chromospheric or aurorae) suggested by a weak $\mathrm{H} \alpha$ detection. This result is similar to the findings on the radio loud M8.5 dwarf TVLM513-46546 (Littlefair et al, 2008). TVLM513 shows $i^{\prime}$ and $g^{\prime}$ band variability in antiphase, initially suggesting the variability is due to patchy dust clouds coupled with the object's fast rotation. However, more recent results show that the $g^{\prime}$ and $i^{\prime}$ bands are no longer correlated. The $g^{\prime}$ band variability has remained stable, but the $i^{\prime}$ band lightcurve has evolved. However, the optical continuum variability is in phase with the $\mathrm{H} \alpha$ flux, again suggesting some magnetic processes are also occurring (Metchev et al, 2013).

The first T dwarf to be confirmed as variable was the T2.5 dwarf SIMP J013656.5 +093347 (Artigau et al, 2009) which was determined to be variable at the $50 \mathrm{mmag}$ level in the $J$ band. A rotation period of 2.4 days was determined from the lightcurves. More interestingly, the lightcurve evolves from night to night (Fig. 2 in Artigau et al (2009)), perhaps due to evolving features such as storms. Radigan et al (2012) studied a similar object, the T1.5 dwarf 2MASS2139, and again found evidence of an evolving light curve in the $J$, and $K_{s}$ wavebands, which after extensive analysis they attributed to heterogeneous clouds with regions of higher opacity. In general, these objects exhibit variable lightcurves, modulated as the object rotates, which evolve on a period of hours (Apai et al, 2013a), days (e.g. Artigau et al 2009; Radigan et al 2012) or even years (Metchev et al, 2013).

While the majority of studied $\mathrm{T}$ dwarfs lie at the L-T dwarf transition region, some late T dwarfs ( $>\mathrm{T} 5$ ) have also been determined to be variable (Clarke et al, 2008). Initially, these atmospheres were thought to be relatively cloud-free, however recent work by Morley et al (2012) suggests that sulphide clouds may exist there (see Sect. 3.4). Buenzli et al (2012) studied one such object and determined a phase offset between different wavelengths while sinusoidal variability was present on the rotation prior of the object. This phase shift is directly linked to the pressure (and hence cloud constituents) probed at each of the observed wavelengths (Marley et al, 2010; Morley et al, 2014a). The lower pressure regions (high altitude) cause the highest phase lag with respect to the highest pressure layers (lowest altitude) and may be as large as half a rotation period. The authors attribute this lag to a change in the opacities (gas or cloud) without a change in the temperature profile, a change in the temperaturepressure profile without a change in the opacity or a combination of the two resulting in a "stacked-cell" atmosphere as seen in Saturn (e.g. Fletcher et al 2011). Robinson and Marley (2014) interpret the phase lag in the frame of their 1D model as being due to thermal fluctuations. However, their model is unable to reproduce the variability on a timescale of $\sim$ hours, as seen in the observations, claiming that a 3D model will be required to explore the dynamics fully.

An alternative to ground based observations is to move into space, minimising differential refraction and atmospheric effects. One of the largest space based vari- 
ability surveys to date was performed by Buenzli et al (2014) who studied 22 brown dwarfs ranging from L5 to T6 with HST. Six of these objects are determined to be variable with another 5 marked as potentially variable. This survey was not sensitive to objects with long periods, but it still suggests that the majority of brown dwarfs have patchy atmospheres, and that there is no spectral type dependence on the fraction of dwarfs that are variable.

Perhaps one of the best studied variable objects is Luhman 16B. Luhman 16AB is the third closest system to the sun at only 2pc (Luhman, 2013) being a L7.5 and T0.5 dwarf binary. Gillon et al (2013) reported variability on a 4.87 hour period with strong night to night evolution which was attributed to a fast evolving atmosphere on the cooler T0.5 dwarf. The L7.5 dwarf is not found to be variable. Burgasser et al (2014) performed spectral monitoring of the system, modelling the T dwarf using a two-spot model and inferring a cold covering fraction of $\sim 30-55 \%$ varying by $15-30 \%$ over a rotation period. This resulted in a difference of $\sim 200-400 \mathrm{~K}$ between the hot and cold regions. Burgasser et al (2014) interpreted the variations in temperature as changes in the covering fraction of a high cloud deck resulting in cloud holes which expose the deeper, hotter cloud layers. They also suggested the rapidly evolving atmosphere may produce winds as high as $1-3 \mathrm{kms}^{-1}$ which is consistent with an advection timescale of 1-3 rotation periods. A new analysis of this system, was produced by Crossfield et al (2014) who used Doppler imaging techniques to produce a global surface map, sensitive to a combination of $\mathrm{CO}$ equivalent width and surface brightness, of the $\mathrm{T}$ dwarf Luhman 16B. The map shows a large, dark mid-latitude region, a brighter area on the opposite hemisphere located near the pole and mottling at equatorial latitudes (Fig. 2 in Crossfield et al 2014). The authors interpreted the map in one of two ways. Either the darker areas represent thicker clouds, obscuring the hotter, inner regions of the atmosphere, and the bright regions correspond to cloud holes providing a view of this warmer interior, or the map shows a combination of surface brightness and chemical abundance variations. They predict that the high latitude bright spot could be similar to polar vortices seen in solar system giant planets, in which case it should be seen in future mapping of this object.

Another class of brown dwarfs that shows photometric variability are those in close $(<10 \mathrm{hrs})$ detached binary systems with white dwarfs (WD0137-349, WD+L6L8, P=116 min: Maxted et al 2006; Burleigh et al 2006; GD1400, WD+L6, P=9.98hrs Farihi and Christopher 2004, Dobbie et al 2005: Burleigh et al 2011; WD0837+185, WD+T8, P=4.2hrs: Casewell et al 2012; NLTT5306, WD+L4-L7, P=101.88 min: Steele et al 2013; CSS21055, WD+L, $\mathrm{P}=121.73$ min: Beuermann et al 2013). These systems are likely tidally locked and as a result one side of the brown dwarf is continually heated by its much hotter white dwarf companion. Three of the five known systems show variability in optical wavelengths at the $1 \%$ level. One of these objects, WD0137-349 has been studied extensively in the near-infrared (Casewell et al, 2013). The white dwarf in this system is 10 times hotter than the brown dwarf, resulting in a difference in brightness temperature of $\sim 500 \mathrm{~K}$ between the day and night sides, likely causing vigorous motion and circulation in the atmosphere (e.g. Showman and Kaspi 2013a). While the substellar objects in these systems are brown dwarfs, and not extrasolar planets, their atmospheres behave in a similar way, both absorbing the (mainly) ultraviolet emissions from their host, and also reflecting the incident light. 
There are brown dwarfs known in close orbits with main sequence stars that are also irradiated (e.g. WASP-30b: Anderson et al 2011, Kelt-1b: Siverd et al 2012), but as their host stars are much more luminous, and the brown dwarf atmosphere scale height is too small to allow transmission spectroscopy, these are much more challenging to observe. 


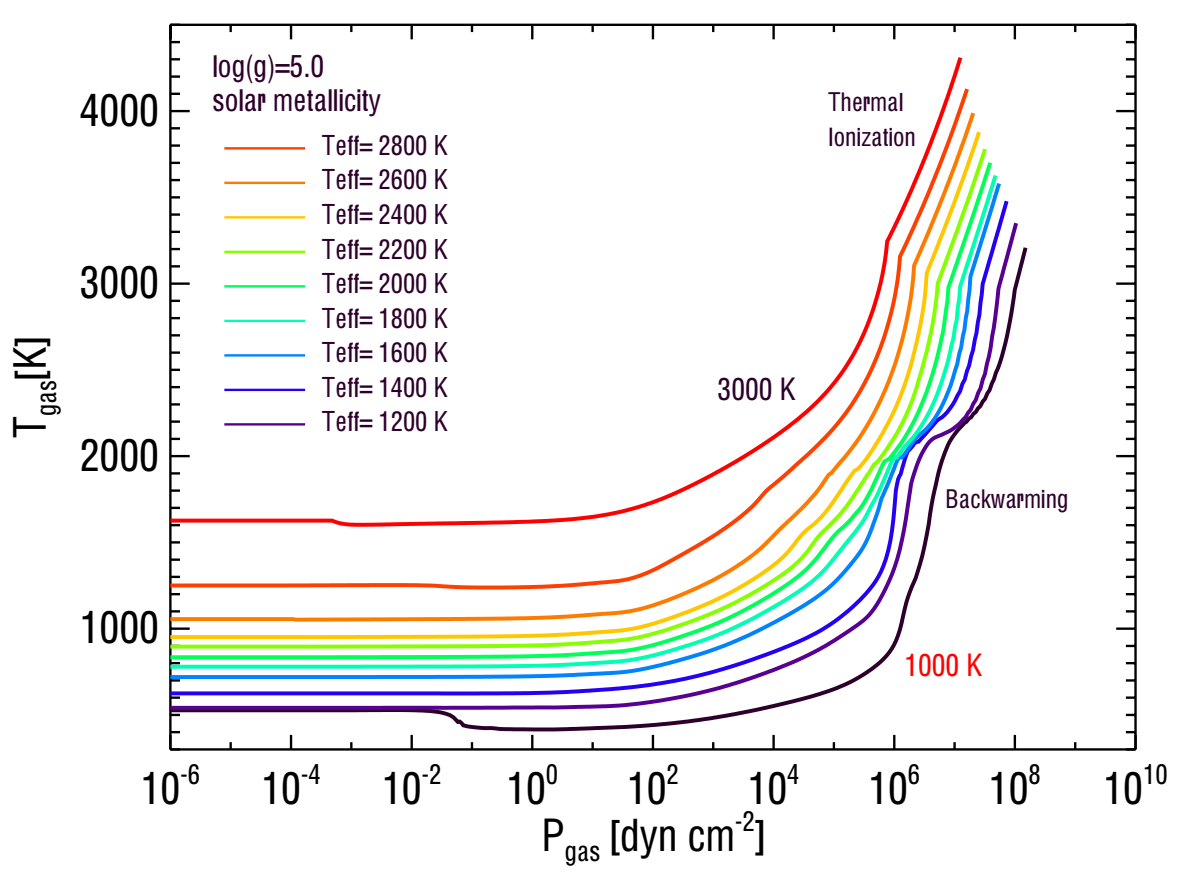

Fig. 5 The local temperature-pressure $\left(T_{\text {gas }}-p_{\text {gas }}\right)$-structure of brown dwarfs $(\log (g)=5.0)$ for effective temperature $\mathrm{T}_{\text {eff }}=1000 \ldots 3000 \mathrm{~K}$. Shown are results from DRIFT-PHOENIX model atmosphere simulations for solar element abundances (Witte et al 2009 2011) [courtesy: Isabel Rodriguez-Barrera]

\section{Theory of brown dwarf atmospheres}

Most, if not all, observational findings reported in the previous sections result from or are influenced by atmospheric processes. The following section will therefore summarize the physics as we expect it to occur in ultra-cool atmospheres of brown dwarfs, including planets and M-dwarfs (Sect. 3.1). Special emphasis will be given to cloud formation processes and their modelling. Section 3.4 contains a summary of different approaches to treat cloud formation as part of model atmospheres.

\subsection{The brown dwarf atmosphere problem}

The atmosphere of a brown dwarf is composed of a cold gas with temperatures $\approx 3000 \mathrm{~K} \ldots<500 \mathrm{~K}$, generally decreasing outwards due to the upper atmosphere being an open boundary by extending into space (Fig. 5). Local temperature inversions could occur due to locally heating processes: A locally increased opacity could lead to radiative heating (e.g. backwarming, see Fig. 5) or cooling, Alfvén wave propaga- 
tion could cause the occurrence of chromospheric structures, and irradiation would provide an additional, external flux source.

The description of the atmosphere of a brown dwarf requires to model the local thermodynamic $\left(\mathrm{T}_{\text {gas }}[\mathrm{K}], \mathrm{p}_{\text {gas }}\left[\mathrm{dyn} / \mathrm{cm}^{2}\right]\right)$, hydrodynamic $\left(\mathrm{v}_{\text {gas }}[\mathrm{cm} / \mathrm{s}], \rho_{\mathrm{gas}}\left[\mathrm{g} / \mathrm{cm}^{3}\right]\right)$ and chemical properties $\left(\mathrm{n}_{\mathrm{x}}\left[\mathrm{cm}^{-3}\right], \mathrm{x}\right.$ - chemical species (ions, atoms, molecules, cloud particles)) in order to predict observable quantities based on the radiative flux $\mathrm{F}_{\lambda}\left[\mathrm{erg} / \mathrm{s} / \mathrm{cm}^{2} / \AA\right]$. The goal is to perform this task by using a minimum of global quantities that are observationally accessible like the resulting total radiative flux $\mathrm{F}_{\text {tot }}=\int F_{\lambda} d \lambda$ through the atmosphere. The classical 1D model atmosphere problem (e.g. Mihalas 1982) is determined by the effective temperature $\mathrm{T}_{\text {eff }}\left(\mathrm{F}_{\text {tot }}=\sigma T_{\text {eff }}^{4}\right.$, $\sigma$ [erg cm $\left.{ }^{-2} \mathrm{~s}^{-1} \mathrm{~K}^{-4}\right]-$ Stefan-Bolzmann constant with the luminosity $L=4 \pi R^{2} \sigma T_{\text {eff }}^{4}$ and the radius $(R[\mathrm{~cm}]))$, the surface gravity $\log (\mathrm{g})\left(\log (g)=\log \left(G M / R^{2}\right), G\left[\mathrm{~cm}^{3} \mathrm{~g}^{-1}\right.\right.$ $\mathrm{s}^{-2}$ ] - gravitational constant, $\mathrm{M}$ - object mass) and the element abundances of the object. Material quantities like the equation of state, and further chemistry and opacity data close this system of equations. Brown dwarf atmosphere models (Lunine et al 1986; Burrows et al 1989;: Tsuji et al 1996a; Tsuji 2002; Ackerman and Marley 2001 . 2013, Allard et al 2001; Burrows et al 2002, Gustafsson et al 2008; Witte et al 2009 2011; for earlier references on M-dwarfs see Allard et al 1997) that are widely applied to optical and IR observations (Sects.2.1.2.3)

- calculate the local gas temperature by solving the radiative and convective energy transport through a stratified medium in local thermal equilibrium (LTE) with an open upper boundary,

- assume flux conservation according to a total energy given by $T_{\text {eff }}$

$\left(F_{\text {tot }}=F_{\text {rad }}+F_{\text {conv }}=\sigma T_{\text {eff }}^{4}\right)$,

- calculate local gas-phase composition for a set of element abundances to determine the opacity of the local gas (Mostly, chemical equilibrium is assumed, in some simulations, $\mathrm{CO} / \mathrm{CH}_{4} / \mathrm{CO}_{2}$ and $\mathrm{N}_{2} / \mathrm{NH}_{3}$ are treated kinetically to better fit observed spectra.),

- calculate the local gas pressure assuming hydrostatic equilibrium.

Brown dwarfs deviate from the classical model atmosphere (e.g. textbook Mihalas 1982) in that clouds form inside their atmospheres. During the formation process, elements are consumed resulting in depleted gas-phase opacity sources like $\mathrm{TiO}, \mathrm{SiO}$ and others. The cloud particles are a considerable opacity source which introduces a backwarming effect (see Fig. 55. Convection plays an important role in transporting material into regions that are cool enough for the condensation processes to start or progress (see also Fig. 88. Convection is a mixing mechanisms that can also drive the local gas-phase out of chemical equilibrium if the transport is faster than the chemical reaction towards the local equilibrium state (Noll et al 1997).

So far, the focus of brown dwarf model atmospheres was on one dimensional simulations in the vertical $(z)$ direction. This assumption implies that the brown dwarf atmosphere is homogeneous in the other two dimensions $(x, y)$. Observation of irradiated brown dwarfs (Casewell et al 2012) suggest what is known for irradiated planets since Knutson et al (2009), namely, that global circulation may also occur on brown dwarfs. We note, however, that the driving mechanisms for global circulation are likely being dominated by rotation in brown dwarfs compared to irradiation alone in 
close-in planets (Zhang and Showman 2014). Variability observations of time-scales different than the rotational period (Sect. 2.3p have long been interpreted as a sign for an inhomogeneous cloud coverage of brown dwarfs. To summarize, the aim of building a model for an atmosphere is to understand the interaction between different processes and to calculate quantities that can be compared to experiments or observation. The input quantities are the global properties $T_{\mathrm{eff}}, L_{\star}, \log (g)$, mass or radius, element abundances, plus material constants for gas chemistry, cloud formation and opacity calculations. The output quantities are details of the atmosphere like the local gas temperature, $T_{\mathrm{gas}}\left[K \overline{]}\right.$, the local gas pressure, $p_{\mathrm{gas}}[\mathrm{bar}]$, the local convective velocity, $\mathrm{V}_{\mathrm{conv}}[\mathrm{cm} / \mathrm{s}]$, the local number densities of ions, atoms, molecules, $n_{\mathrm{x}}\left[\mathrm{cm}^{-3}\right]$, local grain size, a [cm], local number of cloud particles, $n_{\mathrm{d}}\left[\mathrm{cm}^{-3}\right]$, local material composition of grains, the cloud extension, and many more details. Directly comparable to observations is the the resulting spectral surface flux $F_{\lambda}$.

3.2 The chemical repository of the atmosphere

The chemical repository of an atmosphere, including atoms, molecules and cloud particles, is determined by the element abundances available throughout the atmosphere. Different wavelengths with different optical depths probe different atmospheric layers with their specific chemical composition. Primordial element abundances, that should be characteristic for a young, hot brown dwarf, are determined by where and when a brown dwarf formed as the interstellar element abundances increase in heavy elements over time (Yuan et al 2011) and may depend on the star formation history of the brown dwarf's birthplace (Henry and Worthey 1999, Cheng et al 2012). The primordial abundances should be preserved in the brown dwarf's interior and below any atmospheric region that could be affected by cloud formation and down-mixing of processed element abundances. Given the long life times of brown dwarfs, we expect element sedimentation inside the brown dwarf core similar to what we know from white dwarfs. The element abundances that determine the spectral appearance of a brown dwarf are processed element abundances and they differ from the primordial values due to the effect of element depletion by cloud formation, element enrichment by cloud evaporation and the convective mixing of such chemically altered element abundances.

The primordial element abundances are almost always assumed to be the solar element abundances or a scaling thereof. These values are inspired by seismological measurements and 3D simulations to fit high-resolution line profiles. The element abundance values determined for the Sun depend a little on the method and/or simulation applied (Pereira et al 2013, see also discussion in Helling et al 2008a). In principle, there is no reason why any star's element abundances should precisely scale with the solar element abundances (Bergemann et al 2014). Allard et al (1997) summarized the chemical composition in brown dwarf atmospheres as the basis of every model atmosphere simulation. 

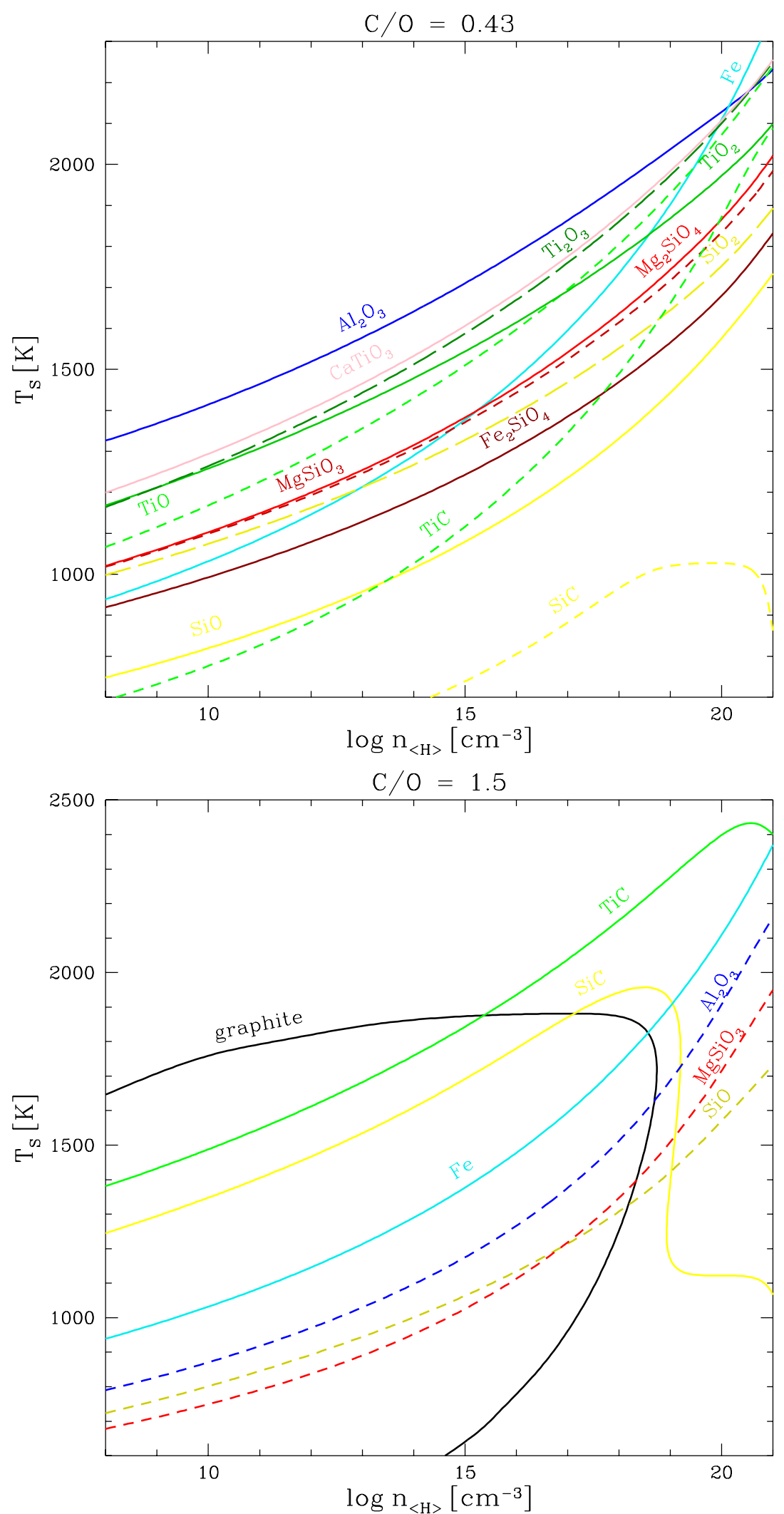

Fig. 6 Thermal stability of various materials in an oxygen-rich $(\mathrm{C} / \mathrm{O}<1$; top $)$ and a carbon-rich $(\mathrm{C} / \mathrm{O}>1$, middle) solar abundance gas. All curves show $\left(T_{\mathrm{gas}}, n_{<H>}\right)$ where the supersaturation ratio $S_{\mathrm{s}}=1$ ( $s=\mathrm{TiO}[\mathrm{s}], \mathrm{Fe}[\mathrm{s}]$, graphite, ...; Eq. 11. The materials will evaporate in the parameter space above each curve. In the case of graphite, the evaporation parameter space is above and to the right of of the curve [courtesy P. Woitke]. 


\subsection{Fundamental ideas on cloud formation}

The following section provides a summary of basic ideas of how clouds form. The formation processes and underlying concepts are based on a microscopic approach which, in the very end, will depend on our quantum-mechanical understanding of chemical reactions leading to more and more complex structures that describe the transition from the gas-phase into the solid or liquid phase. Section 3.4 will summarize different approaches to cloud formation modelling that are applied by different research groups to solve the brown dwarf atmosphere problem.

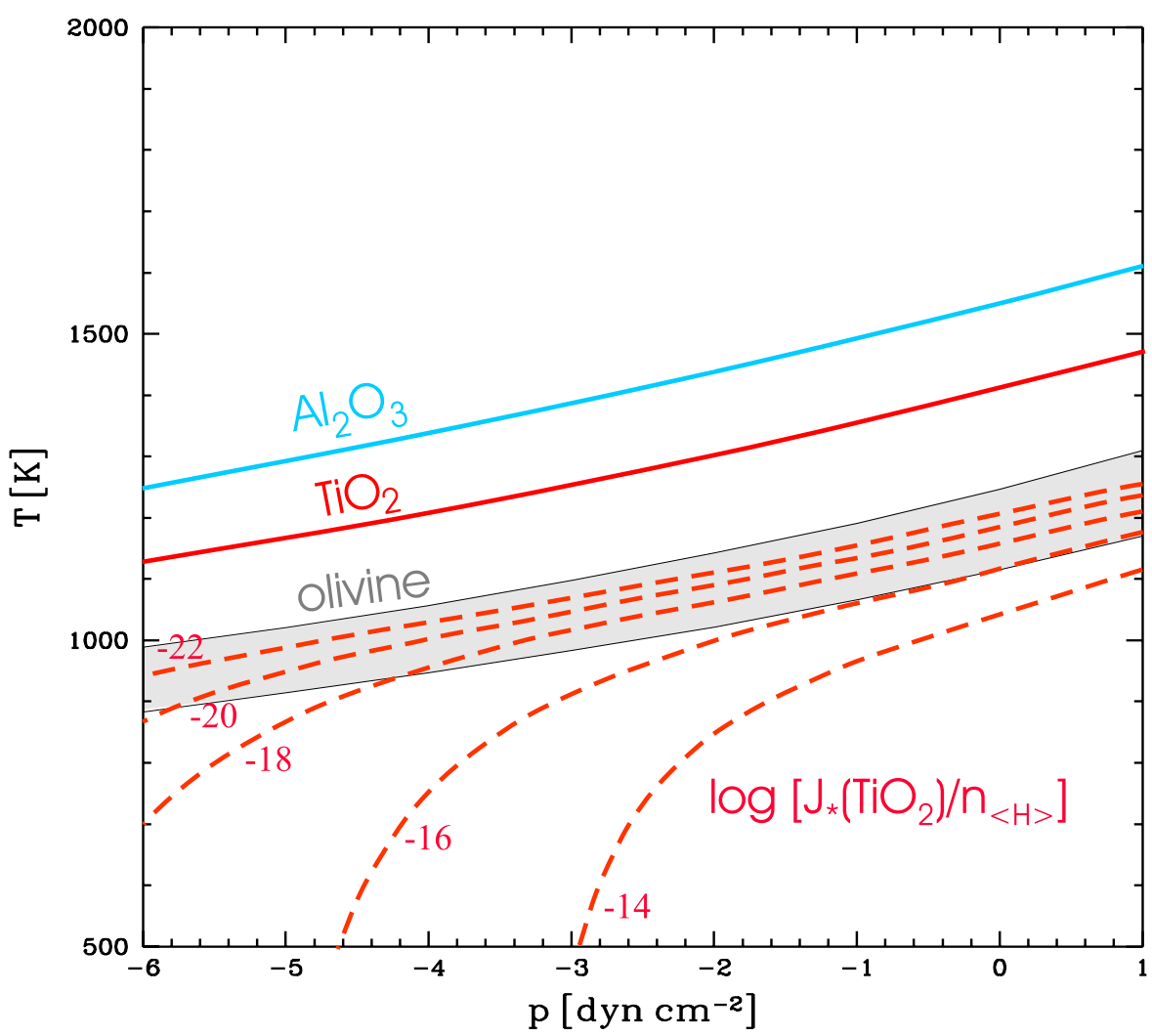

Fig. 7 A considerable supersaturation is required for the seed formation processes to occur. The seed formation rate of $\mathrm{TiO}_{2}$ (nucleation rate $J_{*}$, dashed red line) is highest far below the thermal stability curve for the solid material $\mathrm{TiO}_{2}$ [s] (solid red line). Thermal stability for $\mathrm{Al}_{2} \mathrm{O}_{3}$ [s] and the olivines in a solar abundance gas are shown for comparison. [courtesy P. Woitke]

\subsubsection{Thermal stability}

The concept of thermal stability is used in all but one cloud model to determine if a cloud exists in an atmosphere. Figure 6 shows thermal stability curves usually used 
for this procedure, and which are often called 'condensation curves' in the literature. Such a material is in phase equilibrium which is described by the supersaturation ratio $S_{\mathrm{s}}=1$ of a material $s\left(s=\mathrm{SiO}[\mathrm{s}], \mathrm{TiO}_{2}\right.$ [s], $\mathrm{MgO}[\mathrm{s}], \mathrm{Fe}[\mathrm{s}], \mathrm{Mg}_{2} \mathrm{SiO}_{3}[\mathrm{~s}], \ldots$; [s] referring to 'solid') with the supersaturation ratio being defined as

$$
S_{\mathrm{s}}=\frac{p_{\mathrm{x}}\left(T_{\mathrm{gas}}, p_{\mathrm{gas}}\right)}{p_{\mathrm{sat}, \mathrm{s}}\left(T_{\mathrm{s}}\right)}
$$

$p_{\mathrm{x}}\left(T_{\mathrm{gas}}, p_{\mathrm{gas}}\right)$ is the partial pressure of the growing gas species $x$, and $p_{\mathrm{sat}, \mathrm{s}}\left(T_{\mathrm{s}}\right)$ is the saturation vapour pressure of the solid $s$. The application of the law of mass action to $p_{\mathrm{x}}$ shows that $S_{\mathrm{s}}$ is well-defined no matter whether the monomer of solid $s$ exists in the gas-phase or not (Helling and Woitke 2006). Hence, the concept of thermal stability does not allow to investigate if and how a particular condensate does form. Figures 6 demonstrate below (i.e., $\leqq$ ) which temperatures a material would be thermally stable in an oxygen-rich (top) and a carbon-rich (bottom) environment.

\subsubsection{Cloud formation processes}

Cloud formation (Fig. 8) starts with the formation of condensation seeds in brown dwarfs and giant gas planets where no tectonic processes can provide an influx of dust particles into the atmosphere ${ }^{2}$ This process is a sequence of chemical reactions through which larger molecules form which then grow to clusters and eventually, a small, solid particle emerges from the gas phase. Such reaction chains have been extensively studied in soot chemistry pointing to the key role of PAH (polycyclic aromatic hydrocarbons) in carbon-rich environments (Göres 1993). The modelling aspect of such chemical paths is greatly hampered by cluster data being not always available for a sensible number of reactions steps, and computational chemistry plays an important role for our progress in astrophysics' cloud formation (e.g. Catlow et al 2010). Figure 7 demonstrates that the seed formation process requires a considerable supersaturation of the respective seed forming species: The seed formation rate, $J_{*}$, peaks at a far lower temperature than the thermal stability of the same material suggests. This is very similar to Earth where water vapour condensation on ions requires a supersaturation of $400 \%$. Iraci et al (2010), for example, demonstrate that equilibrium water ice formation is impossible on Mars.

Once condensation seeds have formed, other materials are already thermally stable (Fig. 6) and highly supersaturated (Fig. 1 in Helling et al 2008c). This causes the growth of a substantial mantle via dust-gas surface reactions. The cloud particles forming in an oxygen-rich gas will therefore be made of a mix of all available materials as many materials become thermally stable in a rather narrow temperature interval (top panel, Fig. 6). Once the cloud particles have formed, other intra-particle collision processes may alter the particle size distribution. Such collisions depend on the momentum transfer between particles and may result in a further growth of the

\footnotetext{
2 The formation of weather clouds on Earth involve water condensation of pre-existing seeds particles (condensation nuclei) which origin from volcano outbreaks, wood fires, ocean salt spray, sand storms, and also cosmic-ray-induced ion-ion cluster reactions (see CERN CLOUD experiment). Noctilucent clouds in the upper Earth atmosphere, however, require the recondensation of meteoritic material to understand their existence (Saunders et al 2007).
} 


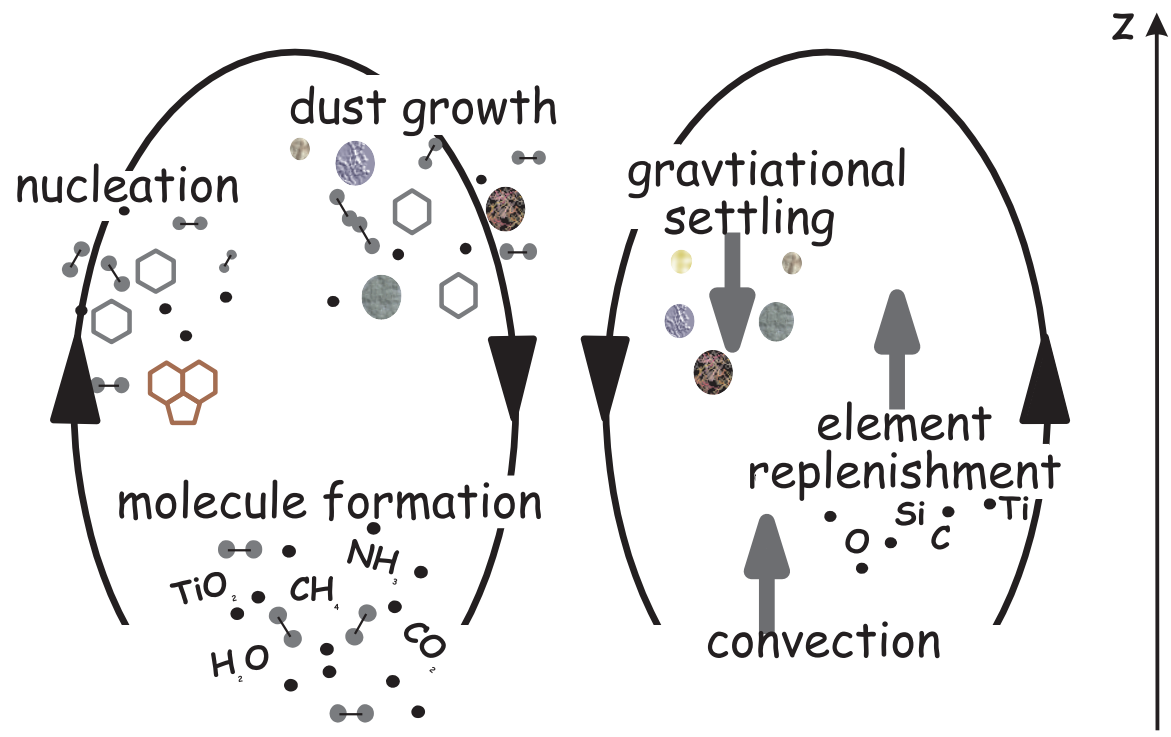

Fig. 8 The circuit of dust that determines cloud formation: cloud particle formation (nucleation, growth) $\rightarrow$ gravitational settling (drift) $\rightarrow$ element depletion \& element replenishment by convective mixing (Woitke and Helling 2004).

particle or in destruction (Güttler et al 2010; Wada et al 2013). Collisions between charged grains may lead to an acceleration of the coagulation process in brown dwarf atmospheres (e.g. Konopka et al 2005).

\subsubsection{Some results on cloud formation}

We use modelling results from Helling \& Woitke to demonstrate the origin of basic cloud properties and feedback mechanisms that determine the formation of clouds. Refined results of a DRIFT-PHOENIX atmosphere simulation (Witte et al 2011) are used for this purpose.

The upper boundary of the cloud is determined by the formation of condensation seeds. Figure 9 demonstrates that a substantial grain growth can only set in when condensation seeds have formed despite of extremely high supersaturation of potentially condensing materials (5th panel). The seed formation rate, $J_{*}\left[\mathrm{~cm}^{-3} \mathrm{~s}^{-1}\right]$ (2nd panel, solid), is calculated for $\mathrm{TiO}_{2}$-nucleation for which cluster data exists (see Helling and Fomins 2013). It determines furthermore the number density of cloud particles, $n_{\mathrm{d}}\left[\mathrm{cm}^{-3}\right]$ (2nd panel, dashed) in the entire cloud. Below a shallow $\mathrm{TiO}_{2}$ [s] layer, all thermally stable materials grow almost simultaneously (3rd and 4th panel) producing core-mantle cloud particles with a mixed mantle composition.

A closer inspection of the material volume fractions $\left(V_{\mathrm{s}} / V_{\text {tot }}\right)$ and the grain growth velocity $\left(\chi_{\mathrm{s}}\right)$ reveals a changing material composition in the cloud with height. The reasons are element consumption and thermal instability: The condensates can not grow further if element depletion causes a sub-saturation, or evaporation sets in if the local temperature is too high. The result is that also the individual supersaturation 


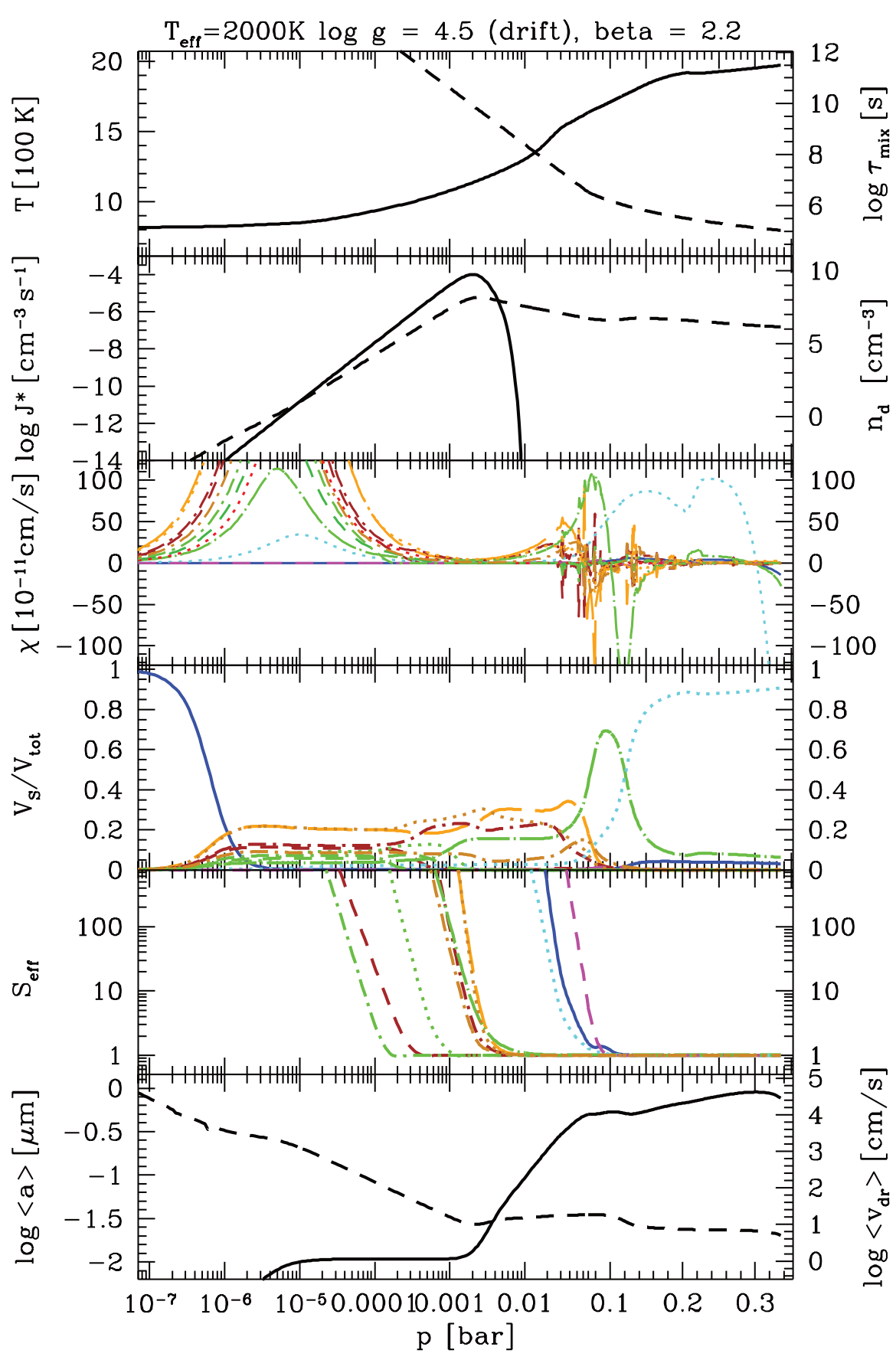

Fig. 9 Cloud model results as part of a DRIFT-PHOENIX atmosphere model $\left(\mathrm{T}_{\text {eff }}=2000 \mathrm{~K}, \log (\mathrm{g})=4.5\right.$, solar metallicity). 1st panel: local gas temperature $T_{\text {gas }}[\mathrm{K}]$ (solid), convective mixing time scale $\tau_{\operatorname{mix}}[\mathrm{s}$ ] (dashed); 2nd panel: seed formation rate $J_{*}\left[\mathrm{~cm}^{-3} \mathrm{~s}^{-1}\right]$ (solid), cloud particle number density $n_{\mathrm{d}}\left[\mathrm{cm}^{-3}\right]$ (dashed); 3rd panel: grain growth velocity $\chi_{\mathrm{s}}\left[\mathrm{cm} \mathrm{s}^{-1}\right]$; 4th panel: material volume fraction $V_{\mathrm{s}} / V_{\text {tot }}[\%]$; 5th panel: effective supersaturation ratio $S_{\text {eff }}$ for each material $s$; 6th panel: mean grain size $\langle a\rangle[\mu \mathrm{m}]$ (solid), drift velocity $\mathrm{v}_{\mathrm{dr}}\left[\mathrm{cm} \mathrm{s}^{-1}\right]$ (dashed). The different colours refer to the same different solid in each of the panels. The subscript $s$ refers to the different condensate materials: $s=\mathrm{TiO}_{2}$ [s] (blue), $\mathrm{Mg}_{2} \mathrm{SiO}_{4}[\mathrm{~s}]$ (orange, long-dash), $\mathrm{SiO}[\mathrm{s}]$ (brown, short-dash), $\mathrm{SiO}_{2}[\mathrm{~s}]$ (brown, dot - short-dash), Fe[s] (green, dot long-dash), $\mathrm{Al}_{2} \mathrm{O}_{3}$ [s] (cyan, dot), $\mathrm{CaTiO}_{3}$ [s] (magenta, dash), $\mathrm{FeO}[\mathrm{s}]$ (green, dash), $\mathrm{FeS}$ [s] (green, dot), $\mathrm{Fe}_{2} \mathrm{O}_{3}[\mathrm{~s}]$ (green, dot - short-dash), $\mathrm{MgO}[\mathrm{s}]$ (dark orange, dot - short-dash), $\mathrm{MgSiO}_{3}$ [s] (dark orange, dot). 


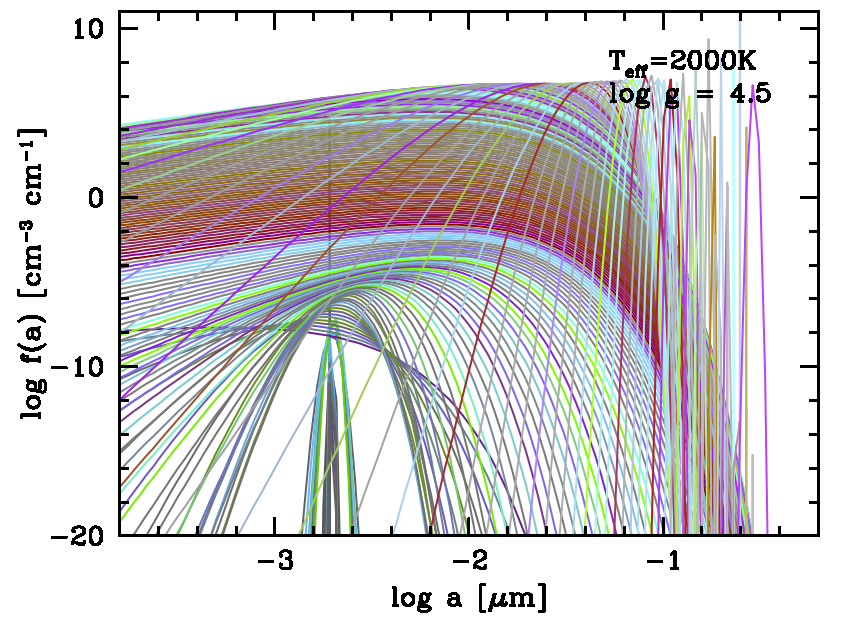

Fig. 10 Grain size distributions, $f(a)\left[\mathrm{cm}^{-3} \mathrm{~m}^{-1}\right]$, differ for different atmospheric layers due to different cloud formation processes contributing and/or dominating. The plot shows the evolution of the grain size distribution from the start of the cloud formation by seed formation (narrow gray-ish peaks), the continues production of seed particles which simultaneously grow (peaks growing in height and moving to the right towards larger grain sizes; blue colours), and the growth dominated distributions (peaks have constant heights and keep moving to the right; purple colours). $f(a)$ is shown for the same atmosphere model like in Fig. 9

ratios approach one, but each at a different temperature (i.e. atmospheric height in 1D models).

Element depletion affects also the seed formation (mainly due to Ti). No more new seed particles can form below a certain height as the increasing temperature hampers the clusters' thermal stability. All cloud particles that exist below this height have rained in from above. These particles fall into a gas of increasing density and temperature. The increasing gas density causes an increasing mean grain size $(\langle a\rangle)$ until the grains fall faster than they can grow. A part of the lower cloud has therefore an almost constant mean grain size. Below that, the temperature is too high and even high-temperature condensates like $\mathrm{Fe}[\mathrm{s}]$ and $\mathrm{Al}_{2} \mathrm{O}_{3}$ [s] evaporate. The thermal stability of the most stable materials determines the cloud's lower boundary. The lower edge of the cloud is made of large particles that consist of very heat-resistant materials like $\mathrm{Al}_{2} \mathrm{O}_{3}[\mathrm{~s}]$ with inclusions of $\mathrm{Fe}[\mathrm{s}]$ and $\mathrm{TiO}_{2}[\mathrm{~s}]$.

\subsubsection{Why do we need a cloud model?}

Important input quantities for model atmosphere simulations, and for retrieval methods, are opacity data for the gas-phase and for the cloud particles. Molecular line lists have been a big issue for a long time (Allard et al 1997, Hill et al 2013). But the calculation of the gas phase absorption coefficient requires also the knowledge of the number density of the absorbing species which is determined by the element abundances of the constituting elements. The element abundances are strongly influenced by how many cloud particles form of which composition and where in the 
atmosphere. A detailed cloud model is therefore needed to calculate how many cloud particles deplete the gas where and of which elements. Helling et al (2008c) detail in their Fig. 7 the changing $[\mathrm{Ti} / \mathrm{H}],[\mathrm{Si} / \mathrm{H}],[\mathrm{Fe} / \mathrm{H}]$, etc abundances with atmospheric height and global parameters when cloud formation is considered.

The cloud opacity is determined by the size distributions of the cloud particles and their material composition as well as the optical constants (refractory index). The cloud opacity changes with height because of the height dependent grain size distribution, but also the material composition of the cloud particles changes with height (Figs. 9, 10). Figure 10 depicts the number of cloud particles for each atmospheric layer considered in the underlying atmosphere model: each atmospheric layer is characterized by one curve. The distribution of the number of particles (denoted by $f(a)$ ) also changes with atmospheric height. The evolution of the cloud particle size distributions is determined by the cloud formation processes summarized in Sect. 3.3.3 The delta-like distributions (dark green curves) are characteristic for the top of the cloud where the nucleation process dominates and surface growth is not yet efficient (compare also panel $2 \& 3$ in Fig. 9). The distributions, $f(a)$, broaden when grain growth becomes efficient and $f(a)$ increases in height when nucleation takes place simultaneously (wide purple curves). This is the case just below the cloud top. Once the size distributions 'move' in grain-size space towards the right (towards larger grain sizes) with a constant peak value, the nucleation has stopped. This is indicative for those cloud regions where the cloud particles rain into deeper atmospheric layers. The deeper cloud layers are characterized by narrow size distributions of large grains as all cloud particles in that layer had time to grow. Eventually, the distribution functions move back into the small-grain region of Fig. 10 because the cloud particles evaporate.

Figure 11 shows wavelength-dependent cloud opacities for individual cloud layers. The silicate absorption features appears clearly in the low-temperature part of the cloud (compare lowest panel Fig. 11p. Scattering dominates the cloud extinction shortward of $4 \ldots 9 \mu \mathrm{m}$ depending on the cloud particle sizes. Hence, for both, the gas opacity and the cloud opacity, a rather detailed cloud model needs to be applied to determine the cloud particle sizes, their size distribution and their material composition depending on the local thermodynamic properties inside the atmosphere.

\subsection{Different approaches to describe cloud formation in atmosphere simulations}

Cloud models are an integral part of each brown dwarf (and planetary) model atmosphere simulation as they determine the remaining element abundances that define the local gas-phase composition of the spectrum forming atmosphere layers. In the following, we summarize the different cloud models that are to date applied and published in model atmosphere simulations of brown dwarfs (and planets and $\mathrm{M}$ dwarfs). This section is an update of Helling et al (2008a) and it includes all brown dwarf cloud models that are part of an atmosphere simulation. Ackerman and Marley (2001) discuss some of the older cloud models (Lunine et al 1986; Rossow 1978). A more planet-focused review is provided in (Marley et al, 2013). Lunine et al (1986) and in their next paper Burrows et al (1989) were the first to introduce a cloud opac- 

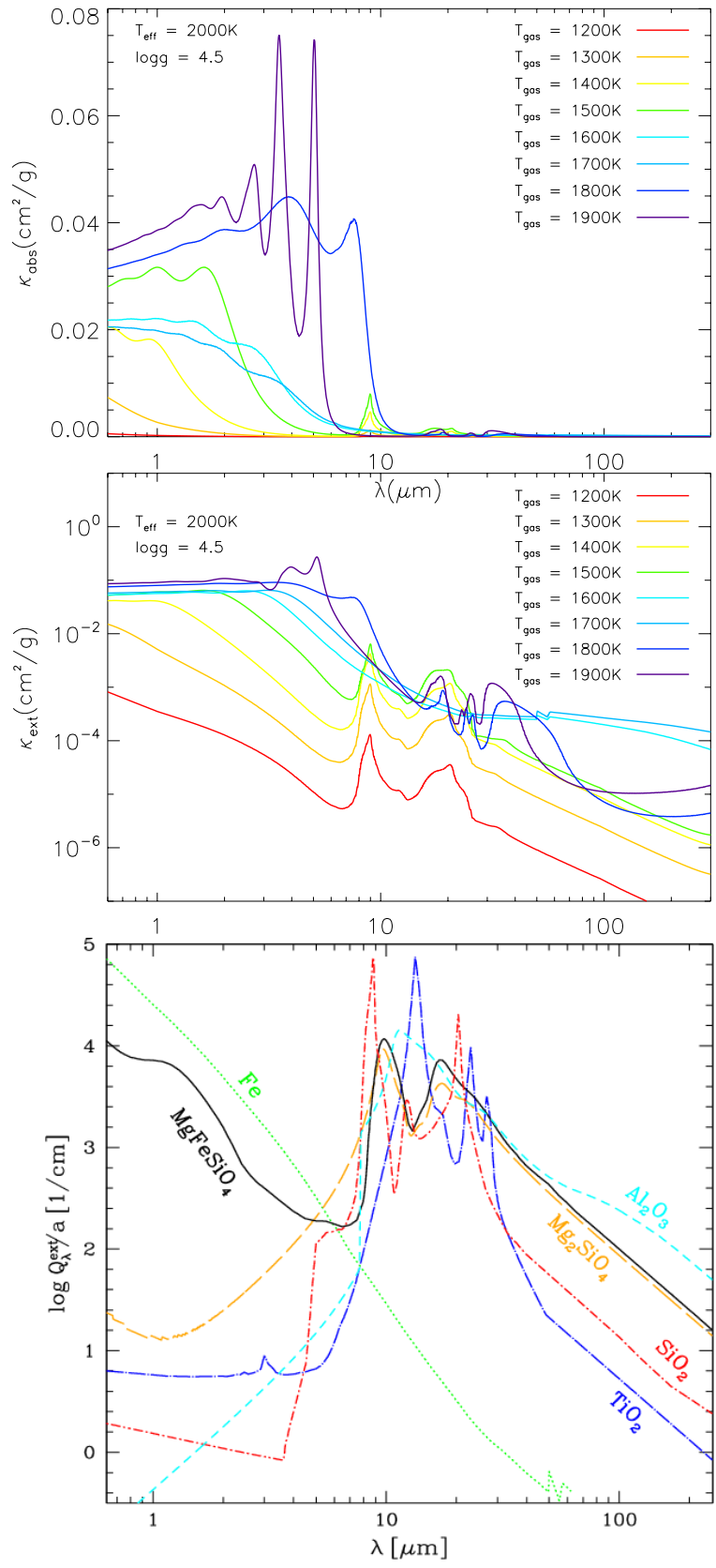

Fig. 11 Top two: Cloud opacity, $\kappa_{\text {abs }}^{\text {dust }}(\lambda)$ and $\kappa_{\text {ext }}^{\text {dust }}(\lambda)=\kappa_{\text {abs }+ \text { scat }}^{\text {dust }}(\lambda)\left[\mathrm{cm}^{2} \mathrm{~g}^{-1}\right]$, as function of wavelength, $\lambda[\mu \mathrm{m}]$, for different layers for a warm brown dwarf $\left(\mathrm{T}_{\text {eff }}=2000 \mathrm{~K}, \log (\mathrm{g})=4.5\right)$. Different colours indicate different layers in the DRIFT-PHOENIX model atmosphere with each layer having different grain sizes and material compositions (Figs. 910 [courtesy: Diana Juncher]. Bottom: Extinction efficiency as function wavelength in the small-particle limit of the Mie-theory (Woitke 2006). 
ity (or 'particulate opacity sources') into their atmosphere model which served as outer boundary for brown dwarf evolution models. Tsuji et al (1996a) suggested that dust needs to be taken into account as opacity source for atmosphere models with $\mathrm{T}_{\text {eff }}<2800 \mathrm{~K}$.

The following cloud models are very different from any cloud parametrization used in the classical retrieval methods. Retrieval methods only use a radiative transfer code that is iterated until an externally given set of parameters (local properties like molecular abundances and gas temperatures) allows to fit a set of observed properties (Madhusudhan and Seager 2009, Benneke and Seager 2012, Barstow et al 2013, Lee et al 2013). Different approaches are used to decide with which quality the parameter set fits the observation. Lee et al (2013) and Line et al (2014) assume Gaussian error for single-best fit solutions, Benneke and Seager (2012) derive a full probability distribution and credibility regions for all atmospheric parameters. Both methods require a prior to start the best-fit procedure in a multi-parameter space to ensure that the global minimum can be found.

i) Tsuji model: Tsuji (2001) suggested that condensates in cool dwarf atmospheres are present in the form of layers with strict inner and outer boundaries. The inner boundary, associated with a certain temperature denoted by $T_{\text {cond }}$, is related to the thermodynamical stability of the cloud particles in the surrounding gas. The upper boundary, parametrized by $T_{\mathrm{cr}}$, is related to the assumption that the cloud particles must remain extremely small, because if they would grow too large then they would otherwise settle gravitationally. For $T_{\text {cond }}>T>T_{\mathrm{cr}}$, the particles are assumed to be constantly forming and evaporating, thereby circumventing the problem of the gravitational settling (Tsuji 2001, 2002; Tsuji et al 2004; Tsuji 2005). Yamamura et al (2010); Tsuji et al (2011); Sorahana et al (2013) have applied their UNIFIED Cloudy MODEL to the unique AKARI data set for brown dwarfs. Sorahana et al (2014) interpreted the mismatch with these models as signature of chromospheric activity on brown dwarfs (see Sect.2.2.2.

ii) Burrows et al. model: Cooper et al (2003) (also Burrows et al 2006) assume chemical and phase equilibrium to determine whether cloud particles of a certain kind are thermodynamically stable in a solar composition gas. If $p_{\mathrm{x}}\left(T_{\mathrm{gas}}, p_{\mathrm{gas}}\right)=p_{\mathrm{sat}, \mathrm{s}}\left(T_{\mathrm{gas}}\right)$, i.e. the material $s$ is thermally stable, the mean size of the particle of a certain homogeneous composition $s$ is deduced from local time-scale arguments (Rossow 1978), considering growth, coagulation (also named coalescence), precipitation and convective mixing. $p_{\mathrm{x}}\left(T_{\mathrm{gas}}, p_{\mathrm{gas}}\right)=p_{\text {sat } \mathrm{s}}\left(T_{\mathrm{gas}}\right)$ also determines the altitude of cloud layers of composition $s$. The amount of dust is prescribed by a free parameter $S_{\max } \approx 1.01$ (maximum supersaturation) which is the same for all materials. Thereby, the supersaturation ratio of the gases is fixed throughout the atmosphere and the mass of cloud particles present in the atmosphere scales with the saturation vapour pressure $p_{\text {sat,s }}(T)$, which decreases exponentially with decreasing $T_{\text {gas }}$. Consequently, the vertical cloud structure is a dust layer with a strict lower boundary and an exponentially decreasing dust-to-gas ratio above the cloud base. Burrows et al (2011) use this phase-equilibrium approach to search where $S_{\mathrm{s}}=1.0$ for individual materials $s$. A 
cloud density function is distributed around this local pressure parametrizing the geometrical cloud extension. For each condensate $s$ the vertical particle distribution is approximated by a combination of a cloud shape function and exponential fall-offs at the high- and low-pressure ends. The model has been used, for example, in Apai et al (2013b) to suggest the presence of an upper, warm thick cloud and a lower, cool thin cloud as reason for the observed atmospheric variability of two early L/T-transition dwarfs (2M2139, SIMP0136; see Sect 2.3). Different cloud shape functions are tested with constant vertical distribution of particles above the cloud base (B-clouds, same as DUSTY models in Allard et al. 2001), and the modal grain sizes per material are adjusted to provided the best spectral fit.

iii) Marley et al. model: Ackerman and Marley (2001) parametrize the efficiency of sedimentation of cloud particles relative to turbulent mixing through a scaling factor, $f_{\text {sed }}$. Large values of $f_{\text {sed }}$ describe rapid particle growth and large mean particle sizes. In this case, sedimentation is efficient, which leads to geometrically and optically thin clouds. When $f_{\text {sed }}$ is small, particles are assumed to grow more slowly and the amount of condensed matter in the atmosphere is larger and clouds are geometrically more extended. Marley et al. solve a diffusion equation that aims to balance the advection and diffusion of each species vapor, $p_{\mathrm{x}}\left(T_{\mathrm{gas}}, p_{\mathrm{gas}}\right)$, and condensate, $p_{\text {sat,s }}\left(T_{\text {gas }}\right)$, at each layer of the atmosphere. It balances the upward transport of vapor and condensate by turbulent mixing with the downward transport of condensate by sedimentation. The downward transport of each condensate is parametrized by $f_{\text {sed }}$ and the turbulent mixing by an eddy diffusion coefficient, $K_{\mathrm{zz}}\left[\mathrm{cm}^{2} \mathrm{~s}^{-1}\right]$. The partial pressure of each condensate species, $p_{\mathrm{x}}$, is compared with the condensate vapour pressure, $p_{\text {sat, } \mathrm{s}}$, the crossing of which defines the lower boundary of the atmosphere above which this particular condensate is thermally stable. The model, as all other aforementioned models, assumes that each material can form by homogeneous condensation. Ackerman and Marley (2001) compute a single, broad log-normal particle size distribution for an assumed modal size that is intended to capture the likely existence of a double-peaked size distribution. Fortney et al (2008) apply this cloud model to planetary atmosphere simulations and, for example, suggest two classes of irradiated planets. Morley et al (2012) applied the Marley et al. model to suggest the presence of an additional cloud layer by moving higher into the atmosphere towards lower temperatures.

iv) Allard et al. model: Phase equilibrium between cloud particles and gas is also assumed in this model (Allard et al 2001). This assumption, i.e. $p_{\mathrm{x}}\left(T_{\text {gas }}, p_{\text {gas }}\right)=$ $p_{\text {sat,s }}\left(T_{\text {gas }}\right)$, is used to determine the cloud base for each condensate individually. Using the time scale for condensation, sedimentation and coalescence (Rossow 1978) in comparison to a prescribed mixing time-scale allows to determine a local mean grain size for a given grain size distribution (Allard et al 2013). The mixing-time scale described the convective overshooting based on a mass exchange frequency guided by Ludwig et al (2002). The BT-SETTL models were applied to learn about variability in brown dwarf atmospheres, for example on Luhman 16 by Crossfield et al (2014). 


\begin{tabular}{|c|c|c|c|c|c|}
\hline & $\begin{array}{l}\text { grain } \\
\text { size }\end{array}$ & $\begin{array}{l}\text { grain } \\
\text { composition }\end{array}$ & $\begin{array}{l}\text { gas } \\
\text { saturation }\end{array}$ & \multicolumn{2}{|c|}{$\begin{array}{l}\text { fitted } \\
\text { parameters }\end{array}$} \\
\hline \multicolumn{6}{|c|}{ Model atmosphere simulations } \\
\hline $\operatorname{Tsuji}\left({ }^{1}\right)$ & $a=10^{-2} \mu \mathrm{m}$ & homog. & $S=1$ & UCM & $\begin{array}{l}\text { dust between } \\
T_{\mathrm{cr}}<T<T_{\text {cond }}\end{array}$ \\
\hline Allard \& Homeier $\left({ }^{2}\right)$ & $\begin{array}{l}f(a)=a^{-3.5} \\
\text { time scales dep. }\end{array}$ & $\begin{array}{l}\text { homog. } \\
\text { homog. }\end{array}$ & $\begin{array}{l}S=1 \\
S=1.001\end{array}$ & $\begin{array}{l}\text { dusty } \\
\text { cond } \\
\text { settl } \\
K_{\mathrm{zz}}\end{array}$ & $\begin{array}{l}\text { full dusty model } \\
\text { dust cleared model } \\
\text { time scales } \\
\text { mixing for non- } \\
\text { equilibrium molecules }\end{array}$ \\
\hline Cooper, Burrows et al. $\left({ }^{3}\right)$ & $\begin{array}{l}f(a) \sim\left(\frac{a}{a_{0}}\right)^{6} \\
\times \exp \left[-6\left(\frac{a}{a_{0}}\right)\right]\end{array}$ & homog. & $S=1.001$ & & $\begin{array}{l}\text { dust between } \\
P_{\text {upper }}^{\text {cloud }}, P_{\text {lower }}^{\text {cloud }}\end{array}$ \\
\hline $\operatorname{Barman}\left({ }^{4}\right)$ & log-norm. $f\left(a, a_{0}\right)$ & homog. & $S=1$ & $P_{\min }, a_{0}$ & \\
\hline Ackerman \& Marley $\left({ }^{5}\right)$ & log-norm. $f(a, z)$ & homog. & $S=1$ & $\begin{array}{l}f_{\text {sed }} \\
K_{\mathrm{zz}}\end{array}$ & $\begin{array}{l}\text { sedimentation } \\
\text { mixing for non- } \\
\text { equilibrium molecules }\end{array}$ \\
\hline Helling \& Woitke $\left({ }^{6}\right)$ & $f(a, z)$ & mixed & $S=S(z, s)$ & & \\
\hline
\end{tabular}

Retrieval method (radiative transfer only + fit quality assessment)

\begin{tabular}{|c|c|c|c|c|}
\hline $\begin{array}{l}\text { Barstow, Irwin, Fletcher }\left({ }^{7}\right) \\
+ \text { Benneke \& Seager }\left({ }^{8}\right)\end{array}$ & $\begin{array}{l}a_{1} \\
\log \text {-norm. } f\left(a_{2}, z\right)\end{array}$ & homog. & - & $\begin{array}{l}\mathrm{n}_{\text {mix }}\left(\mathrm{H}_{2} \mathrm{O}, \mathrm{CO}_{2}, \mathrm{CH}_{4}\right) \\
\left.\tau^{\text {loud }}\left(a_{1}, a_{2}\right), \mathrm{R}_{\mathrm{Pl}} @ 10 \text { bar }\right)\end{array}$ \\
\hline $\begin{array}{l}\text { Lee, Heng \& Irwin }\left({ }^{9}\right) \\
+ \text { Line, Fortney, } \\
\text { Marley, \& Sorahana }\left({ }^{10}\right)\end{array}$ & $a=\mathrm{const}$ & homog. & - & $\begin{array}{l}P_{\mathrm{up}}^{\text {cloud }}, P_{\text {down }}^{\text {cloud }}, \tau^{\text {cloud }}\left(Q_{\text {ext }}(a)\right) \\
\rightarrow \text { more parameter possible }\end{array}$ \\
\hline \multicolumn{5}{|c|}{ 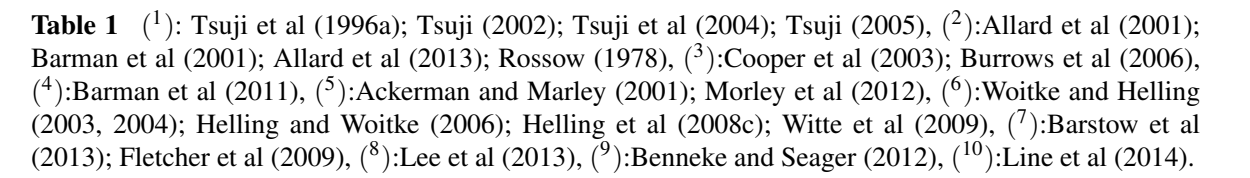 } \\
\hline
\end{tabular}

v) Barman model: Also Barman et al (2011) assume phase-equilibrium and find the lower boundary of the cloud (cloud base) where the atmospheric $\left(T_{\mathrm{gas}}, p_{\mathrm{gas}}\right)$-profile intersects the thermal stability curve of a condensate $\left(p_{\mathrm{x}}\left(T_{\mathrm{gas}}\right)=p_{\text {sat.s }}\left(T_{\mathrm{gas}}\right)\right)$. The particle sizes follow a log-normal distribution with a prescribed modal size, $a_{0}$. The adjustable parameter $a_{0}$ can vary between 1 and $100 \mu \mathrm{m}$ and is the same for each atmospheric height. Prescribing the particle sizes allows to determine the number of cloud particles (equilibrium dust concentration). The cloud height and density above the cloud base is determined by a free parameter $P_{\min }$. The equilibrium dust concentration is assumed if $p_{\text {gas }} \geq P_{\min }$, and decays exponentially for $p_{\text {gas }}<P_{\min }$. If $P_{\min }>p_{\text {sat,s }}\left(T_{\text {gas }}\right)$, then the maximum dusty-to-gas ratio is lowered relative to the equilibrium concentration.

vi) Woitke \& Helling model: This model is different from all above models i)-v) as it kinetically describes seed formation and growth/evaporation coupled to gravitational settling, convective mixing and element depletion by conservation equations following Sect 3.3.2 These intrinsically time-dependent processes (Helling et al 2001; Woitke and Helling 2003) are treated in a stationary approximation of conservation equations (Woitke and Helling 2004; Helling and Woitke 2006, Helling 
et al 2008a) to allow the coupling with a model atmosphere code (DRIFT-PHOENIX, Helling et al 2008b; Witte et al 2009, 2011). The convective mixing with overshooting is parametrized according to a mass exchange frequency (Ludwig et al (2002)). DRIFT-PHOENIX model atmospheres were applied to study early universe, metaldeficient brown dwarfs (Witte et al 2009), and they have recently been used to explore ionization and discharge processes in ultra-cool, cloud-forming atmospheres (Helling et al 2011b a, 2013; Rimmer and Helling 2013, Stark et al 2013.

\section{Model approach summary:}

- All phase-equilibrium models (i-v) assume that each condensate can form by homogeneous condensation, hence, it is assumed that the monomer exist in the gas phase. - All phase-equilibrium models (i-v) adjust their element abundances according to by how much the monomer partial pressure exceeds the vapour pressure $p_{\mathrm{s}}>p_{\mathrm{sat}, \mathrm{s}}$. A prescribed size distribution allows the calculation of the cloud particle sizes. Note that the two prescribed distribution functions (power law and log normal) differ strongly in their dust mass distribution due to the relative contribution of the different sizes.

- All phase-equilibrium models (i-v) are relatively easy to implement.

- Table 3.4 provides a comparison of all cloud models, including free parameters for each cloud model.
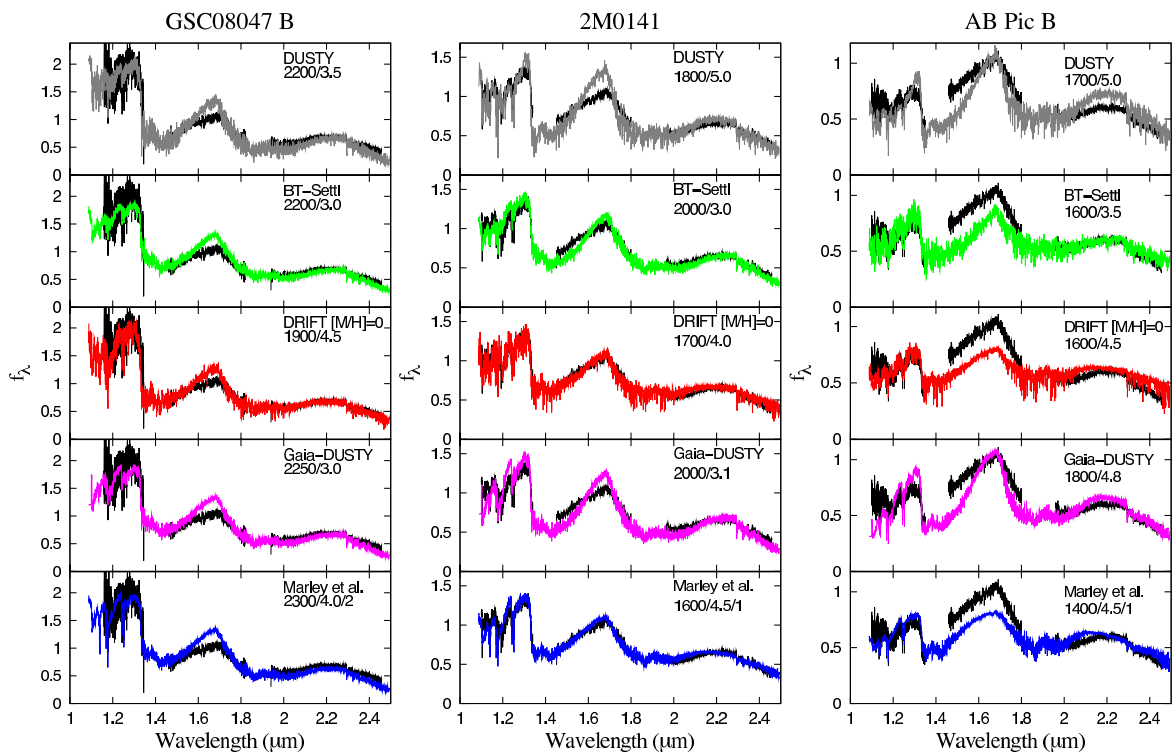

Fig. 12 Patience et al (2012) demonstrate spectral fits for varies Brown Dwarfs with synthetic spectra from different model families. Different model families were also used by Dupuy et al (2010) to provide a good error estimate on the derived global parameters. The figure shows that all model atmospheres found it challenging to fit brown dwarfs at the lowest $\mathrm{T}_{\text {eff }}$ depicted. 


\section{Benchmarking atmosphere models and comparison to observations}

A 'benchmark' is per definition a well-defined test case which is performed to understand differences in different approaches to the same problem. Astronomers and Astrophysicists approach this differently.

The observational astronomy, which aims on benchmarking brown dwarf models, follow the original idea that was inspired by stellar binary systems for which one companion's parameters are well known (Pinfield et al 2006; Burningham et al 2013). 'Benchmark brown dwarfs' are objects for which the distance (and thus luminosity), the age and the metallicity can be determined from observations. Their radii and their mass can be derived from evolutionary models if the age is well constrained. The effective temperature and the surface gravity can now be determined from the luminosity and the radius, the mass and radius, respectively. With $\mathrm{T}_{\text {eff }}, \log (\mathrm{g})$ and a known metallicity, hence known element abundances, a model atmosphere is well constrained and a synthetic spectrum can be produced. This model spectrum is now compared to the binary target. Obvious places to search for such benchmark objects are open clusters as their age, composition and distance can be well defined through observations of much more luminous cluster members, and near-by kinematic moving groups with well-defined membership, age and composition.

Theoretical astrophysics has two model systems to benchmark in our context of brown dwarfs: the atmosphere models and the evolutionary models. Both model systems are complex. The additional challenge is that both systems are not independent because the model atmospheres serve as outer boundaries during the run of evolutionary models. Benchmarking both systems requires a substantial effort from different research groups world-wide, and it is unlikely to happen in the near future. Ongoing areas of research, however, are dedicated comparison studies that aim to demonstrate the difficulties in using model atmosphere and/or evolutionary grids as 'black boxes' (e.g. Sinclair et al 2010), and to allow the observer community to use an error estimate for model atmospheres (Bozhinova et al 2014) and evolutionary models (Southworth 2009). A sensitive handling of such uncertainties is not only important for our immediate understanding of brown dwarfs, but also for detecting planets around brown dwarfs and M-dwarfs (e.g. Rojas-Ayala et al 2013; Triaud et al 2013.

Model atmosphere test are conducted in various ways. Rajpurohit et al (2012) test different model atmosphere families in finding the stellar parameters for the late M-dwarf LHS 1070. They also including the cloud-free MARCS models in their comparison with the cloud-modelling PHOENIX-families (BTsettl, DRIFT). Dupuy et al (2010) and Patience et al (2012) compare the quality of fits to observations by model atmospheres that describe the same physical problem, a cloud-forming atmosphere (Fig. 12). The authors point out the significant difference in the $H$-band spectrum for the different models. This effects decreases with increasing $\mathrm{T}_{\text {eff }}$ but does still impact the $J$-band for $\mathrm{T}_{\text {eff }}=1700 \mathrm{~K}$. The primary differences is in the cloud modelling. The cloud models differ in details (grain sizes, material composition) that influence the local opacity of the cloud and also of the gas phase through element depletion. For example does a higher cloud opacity results in less flux in the $J$-band as the water abundance decreases and, hence, results in weakened water absorption bands. The approach presented in Dupuy et al (2010) and Patience et al (2012) allows an error 
estimate of the derived, global stellar parameters. A more detailed comparison of various brown dwarf model families is presented in Helling et al (2008a). The focus of this paper is comparing cloud model results for prescribed test cases. This also led to more understanding about why the synthetic spectra from different model families differ and which role the cloud modelling plays.

The Virtual Observatory pioneers the incorporation of grids of different model atmosphere families into their data base. A comparison between different model families can provide estimates of the expected systematic errors, which is of interest for the outcome of space missions. Sarro et al (2013) performed the task of predicting how well $\mathrm{T}_{\text {eff }}$ can be determined from GAIA data for Brown Dwarfs based in the BTSettle model atmosphere grid. The Virtual Observatory is now capable of facilitating a multi-model family approach.

\section{Increasing completeness and increasing model complexity}

It may seem a big leap from a 1D atmosphere code to an atmosphere model that allows the study of time-variable cloud cover, the formation of photochemically driven hydro-carbonaceous macro-molecules, magnetic interactions, and irradiation. All these depend on global parameters like rotational period, magnetic field strength, cosmic ray flux etc. However, several aspects on this list start to emerge in the literature.

\subsection{Multi-dimensional, dynamical atmosphere simulations}

First steps towards a multi-dimensional approach to cloud forming brown dwarf atmospheres were made by $2 \mathrm{D}$ hydrodynamical simulations that treat dust formation (nucleation, growth/evaporation, element depletion), turbulence and radiative heating/cooling (Helling et al 2001, 2004), and by large-scale 2D radiative-convection simulations that included the dust growth/evaporation processes for a prescribed number of nucleation seeds (Freytag et al 2010). Both works suggest that clouds will not be present as a homogeneous, carpet-like layer but that cloud particles form, depending on the local temperature and density field, intermittently resulting in patchy cloud structures. Robinson and Marley (2014) came forward with a similar suggestion of local temperature variations to explain brown dwarf variability. Showman and Kaspi (2013b) use a 3D approach to simulate a globally circulating brown-dwarf atmosphere but excluding cloud opacities, turbulence, and radiation. These authors suggest that a hydrodynamically induced horizontal temperature variation of $\Delta T=50 \mathrm{~K}$ can lead to flux variations of $\Delta F / F \approx 0.02-0.2$. Each of these models addressed a different aspect of a multi-dimensional, dynamical atmosphere simulations. The challenges faced by all simulation is illustrated by comparing the following time scales that are characteristic for interacting processes. The below table demonstrates that it is misleading to consider atmospheric processes as independent from each other, but that for example cloud formation processes could be re-ignited by transport processes like gravitational settling or gas-mixing that provides new condensable material:

radiative cooling $\diamond: \quad \tau_{\text {rad }}\left(\rho_{\text {gas }}\right) \quad=\left(\frac{4 \pi \kappa}{c_{\mathrm{V}}} \frac{\partial B}{\partial T}\right)^{-1}=0.5 \ldots 100$ days 


\begin{tabular}{|c|c|c|}
\hline gravitational settling $^{\dagger}$ : & $\tau_{\text {sink }}\left(a, \rho_{\mathrm{gas}}\right) \quad=\frac{H_{\mathrm{p}}}{v_{\mathrm{drift}}}$ & $\begin{array}{l}\text { (with dust without dust) } \\
=15 \mathrm{~min} \ldots 8 \text { month } \\
(a=100 \mu \mathrm{m} \ldots 0.1 \mu \mathrm{m})\end{array}$ \\
\hline large-scale convection: & $\tau_{\text {conv }}\left(\nabla T_{\text {gas }}\right)$ & $=20 \mathrm{~min} \ldots 3.5 \mathrm{~h}$ \\
\hline diffusive eddy mixing ${ }^{\ddagger}$ : & $=\frac{H_{\mathrm{p}}^{2}}{K_{\mathrm{edd}}}$ & $=3 \mathrm{~h} \ldots 3 \mathrm{yrs}$ \\
\hline grain growth: & $\tau_{\mathrm{gr}}\left(T_{\mathrm{gas}}, \rho_{\mathrm{gas}}\right)=\frac{a}{\mathrm{v}_{\mathrm{gr}}}$ & $=0.1 \mathrm{~s} \ldots 1.5 \mathrm{~min}$ \\
\hline $\begin{array}{l}\text { wave propagation: } \\
\text { seed formation: }\end{array}$ & $\begin{array}{l}\tau_{\text {wave }}\left(T_{\text {gas }}, \rho_{\text {gas }}\right)=\frac{H_{\mathrm{p}}}{u+c_{\mathrm{s}}} \\
\tau_{\text {nuc }}\left(T_{\text {gas }}, \rho_{\text {gas }}\right)=\frac{n_{\mathrm{d}}}{J_{*}}\end{array}$ & $\begin{array}{l}=0.3 \mathrm{~s} \ldots 3 \mathrm{~s} \\
\approx 10^{-3} \mathrm{~s}\end{array}$ \\
\hline
\end{tabular}

$\diamond$ Please refer to Table 1 in Helling et al (2011b for definitions and values of the absorption coefficient $\kappa$, $c_{\mathrm{V}}$ specific heat capacity for constant volume and $B(T)$ the frequency integrated Planck function.

$\dagger$ Please refer to Woitke and Helling (2003 2004) for definition of $\mathrm{v}_{\text {drift }}, \mathrm{v}_{\mathrm{gr}}$, the mean grain size $a$, the number of cloud particles $n_{\mathrm{d}}$, and the seed formation rate $J_{*}$. Typical values for all other quantities are applied, $H_{\mathrm{p}} \approx 10^{6} \mathrm{~cm}$.

${ }_{\text {eddy }}\left[\mathrm{cm}^{2} \mathrm{~s}^{-1}\right]\left(\right.$ or $K_{\mathrm{zz}}$ ) is the eddy diffusion coefficient ranging between $10^{4} \ldots 10^{8} \mathrm{~cm}^{2} \mathrm{~s}^{-1}$, see Bilger et al (2013)

Diffusion, gravitational settling and convection have the longest time scales in a brown dwarf atmosphere compared to chemical timescales for cloud particle nucleation and growth. The wave propagation time scale can be used as proxy for turbulence acting on small scales where chemical processes would take place. The wave propagation takes still $100 \times$ longer, and hence, cloud particle formation would be given too much time if a numerical scheme uses wave propagation to set integration time-steps. This is problematic as the cloud particle formation determines the remaining gas phase abundance which in turn determines the local gas opacity and with that the local gas temperature, and eventually, the energy transport and the spectral flux. The radiative cooling time scale indicates the impact of the radiative energy transport on the local hydrodynamics through the energy equation. Depending on the local opacity, radiative cooling can be very efficient, even causing local gas volumes to implode (Helling et al 2001).

\subsection{Gas-phase non-equilibrium effects}

Deviations from local chemical gas-phase equilibrium in the upper atmosphere are suggested to be caused by a rapid convective and/or diffusive up-mixing of warm gases from deeper atmospheric layers combined with a slow relaxation into chemical equilibrium (Saumon et al 2000). Other processes that drive the local gas-phase out of chemical equilibrium (and LTE) are photodissociation, or ion-neutral chemistry initiated by cosmic ray impact (Rimmer and Helling 2013). The local chemical composition of the atmosphere is derived from extensive gas-phase rate network calculations under the influence of vertical mixing and photodissociation. Most of these studies are performed for irradiated planets (e.g. Moses et al 2011; Venot et al 2012 3 A height in the atmosphere (so-called quenching height) is derived above which the

\footnotetext{
3 Venot et al (2012)'s chemical network is publicly available under http://kida.obs.u-bordeaux1.fr/.
} 
gas kinetic reactions are too slow to considerably change molecular abundances. This idea of modelling time-dependent gas non-equilibrium effects has two shortcomings: First, the diffusive eddy mixing coefficient $K_{\text {eddy }}\left[\mathrm{cm}^{2} \mathrm{~s}^{-1}\right]$ (or $K_{\mathrm{zz}}$ ) becomes an additional fitting parameter for model atmospheres to observations (e.g. Cushing et al 2010). Second, reaction rate coefficients differ in the literature leading differences in destruction time scale for example for $\mathrm{C}_{2} \mathrm{H}_{2}$ and $\mathrm{C}_{2} \mathrm{H}_{6}($ Bilger et al 2013). This is, however, a challenge faced by all gas-kinetic approaches and considerable effort is ongoing to weed-out the respective rates. Rimmer et al (2014) model cosmic ray transport through an brown dwarf atmosphere and they demonstrate how galactic cosmic rays influence the abundance of hydrocarbon molecules through ion-neutral reactions.

\section{Closing the loop}

The first L4 dwarf (GD 165B; Becklin and Zuckerman 1988) was discovered $\sim 30$ years ago. Since then, spectral classification of these very cool objects led to the introduction of the new spectral classes $\mathrm{L}, \mathrm{T}$ and $\mathrm{Y}$, with the $\mathrm{Y}$ dwarfs having $\mathrm{T}_{\text {eff }}$ typical for planets. Such low temperature immediately suggest that brown dwarf atmospheres must contain a chemically very rich gas from which clouds will form. The evolutionary transition from the $\mathrm{L}$ into the $\mathrm{T}$ dwarf spectral type are associated with atmospheric variabilities which is attributed to variable cloud coverage. In parallel to the increasing number of observations, atmosphere modellers adopted stellar atmosphere codes for cooler gases by introducing cloud models and additional gas opacity sources (e.g. $\mathrm{CO}_{2}, \mathrm{CH}_{4}, \mathrm{NH}_{3}$ ). More complex processes like kinetic gas chemistry, turbulence and multi-dimensional hydrodynamic simulations were performed but with far less consistency between the processes. The picture that emerged for a brown dwarf atmosphere is that of chemically very active gas that is exposed to phase-changes, turbulence, high and low energy radiation. It also provides a valuable path towards the understanding for brown dwarfs as planetary host stars (Triaud et al 2013) and of climate evolution on extrasolar planets. More and more similarities to planets arise: Radio and X-ray observations suggest brown dwarf atmospheres to be ionized to a certain extend. Theoretical studies on ionization processes support this idea by demonstrating that clouds in brown dwarfs will be charged (Helling et al 2011a b), that clouds can discharge in form of lighting (Bailey et al 2013), that Cosmic Rays can ionize the upper atmosphere and the upper part of the cloud (Rimmer and Helling 2013, and that hydrodynamic winds can provide a source for gas-ionization (Stark et al 2013). Figure 13 shows that these ionization processes (boxes in figure) do appear with different efficiencies in different parts of the atmosphere, suggesting a brown dwarf atmosphere to be a stratified ionized medium rather than a cold, neutral gas.

\section{References}

1. Ackerman AS, Marley MS (2001) Precipitating Condensation Clouds in Substellar Atmospheres. ApJ556:872-884, DOI 10.1086/321540, astro-ph/0103423 
Non-thermal ionisation processes

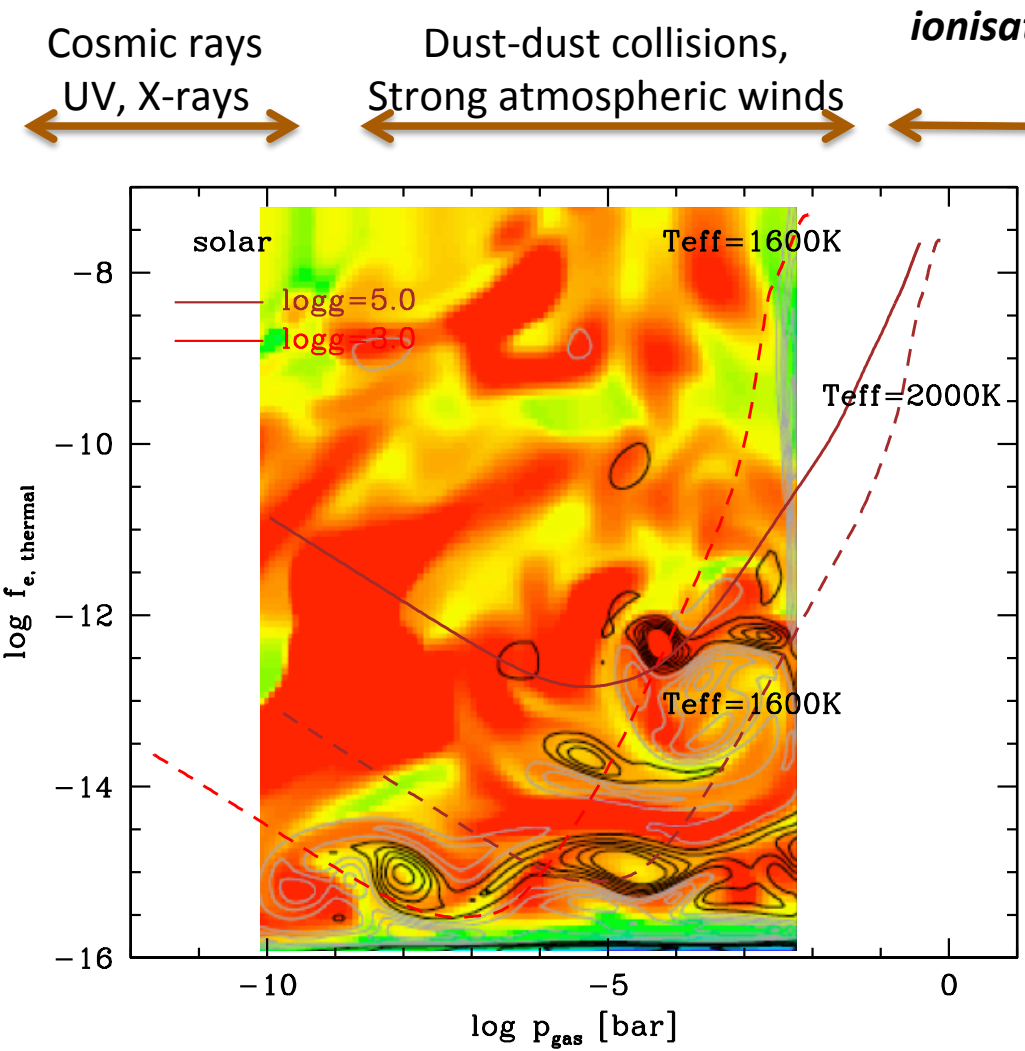

Fig. 13 A brown dwarf atmosphere is not only stratified by its cloud structure (2D colour overlay). Different non-thermal ionization processes occur in brown dwarf atmospheres (boxes: cosmic rays, dust-dust collisions, strong winds) which produce free charges through the atmosphere with varying efficiency. The local degree of thermal ionization (brown solid and dashed lines) is shown in the background for illustration, a 2D simulation of turbulent dust formation indicates where the cloud is located in the atmosphere. (The 2D colour-coded plot contains contour lines of the local vorticity; see Fig 8 in Helling et al (2004))

2. Ackerman AS, Marley MS (2013) Erratum: "Precipitating Condensation Clouds in Substellar Atmospheres". ApJ765:75, DOI 10.1088/0004-637X/765/1/75

3. Allard F, Hauschildt PH, Alexander DR, Starrfield S (1997) Model Atmospheres of Very Low Mass Stars and Brown Dwarfs. ARA\&A35:137-177, DOI 10.1146/ annurev.astro.35.1.137

4. Allard F, Hauschildt PH, Alexander DR, Tamanai A, Schweitzer A (2001) The Limiting Effects of Dust in Brown Dwarf Model Atmospheres. ApJ556:357-372, DOI $10.1086 / 321547$, astro-ph/0104256

5. Allard F, Homeier D, Freytag B, Schaffenberger, W, Rajpurohit AS (2013) Progress in modeling very low mass stars, brown dwarfs, and planetary mass objects. Memorie della Societa Astronomica Italiana Supplementi 24:128, 1302.6559

6. Allers KN, Liu MC (2013a) A Near-infrared Spectroscopic Study of Young Field 
Ultracool Dwarfs. ApJ772:79, DOI 10.1088/0004-637X/772/2/79, 1305.4418

7. Allers KN, Liu MC (2013b) A near-infrared spectroscopic study of young field ultracool dwarfs: additional analysis . Mem. Societa Astronomica Italiana84:1089, 1307.7153

8. Anderson DR, et al (2011) WASP-30b: A $61 \mathrm{M}_{\text {Jup }}$ Brown Dwarf Transiting a V = 12, F8 Star. ApJL726:L19, DOI 10.1088/2041-8205/726/2/L19, 1010.3006

9. Antonova A, Doyle JG, Hallinan G, Golden A, Koen C (2007) Sporadic long-term variability in radio activity from a brown dwarf. A\&A472:257-260, DOI 10.1051/ 0004-6361:20077231, 0707.0634

10. Antonova A, Hallinan G, Doyle JG, Yu S, Kuznetsov A, Metodieva Y, Golden A, Cruz KL (2013) Volume-limited radio survey of ultracool dwarfs. A\&A549:A131, DOI 10.1051/0004-6361/201118583, 1212.3464

11. Apai D, Radigan J, Buenzli E, Burrows A, Reid IN, Jayawardhana R (2013a) HST Spectral Mapping of L/T Transition Brown Dwarfs Reveals Cloud Thickness Variations. ApJ768:121, DOI 10.1088/0004-637X/768/2/121, 1303.4151

12. Apai D, Radigan J, Buenzli E, Burrows A, Reid IN, Jayawardhana R (2013b) HST Spectral Mapping of L/T Transition Brown Dwarfs Reveals Cloud Thickness Variations. ApJ768:121, DOI 10.1088/0004-637X/768/2/121, 1303.4151

13. Artigau É, Bouchard S, Doyon R, Lafrenière D (2009) Photometric Variability of the T2.5 Brown Dwarf SIMP J013656.5+093347: Evidence for Evolving Weather Patterns. ApJ701:1534-1539, DOI 10.1088/0004-637X/701/2/1534,0906.3514

14. Audard M, Osten RA, Brown A, Briggs KR, Güdel M, Hodges-Kluck E, Gizis JE (2007) A Chandra X-ray detection of the L dwarf binary Kelu-1. Simultaneous Chandra and Very Large Array observations. A\&A471:L63-L66, DOI 10.1051/ 0004-6361:20078093,0707.1882

15. Bailer-Jones CAL, Mundt R (2001) Variability in ultra cool dwarfs: Evidence for the evolution of surface features. A\&A367:218-235, DOI 10.1051/0004-6361: 20000416, astro-ph/0012224

16. Bailey RL, Helling C, Hodosán G, Bilger C, Stark CR (2013) Ionisation in atmospheres of brown dwarfs and extrasolar planets VI: Properties of large-scale discharge events. ArXiv e-prints 1312.6789

17. Barman TS, Hauschildt PH, Allard F (2001) Irradiated Planets. ApJ556:885-895, DOI 10.1086/321610, astro-ph/0104262

18. Barman TS, Macintosh B, Konopacky QM, Marois C (2011) Clouds and Chemistry in the Atmosphere of Extrasolar Planet HR8799b. ApJ733:65, DOI 10.1088/ 0004-637X/733/1/65, 1103.3895

19. Barstow JK, Aigrain S, Irwin PGJ, Fletcher LN, Lee JM (2013) Constraining the atmosphere of GJ 1214b using an optimal estimation technique. MNRAS434:2616 2628, DOI 10.1093/mnras/stt1204, 1306.6567

20. Becklin EE, Zuckerman B (1988) A low-temperature companion to a white dwarf star. Nature336:656-658, DOI 10.1038/336656a0

21. Beichman C, Gelino CR, Kirkpatrick JD, Cushing MC, Dodson-Robinson S, Marley MS, Morley CV, Wright EL (2014) WISE Y Dwarfs As Probes of the Brown Dwarf-Exoplanet Connection. ArXiv e-prints 1401.1194

22. Benneke B, Seager S (2012) Atmospheric Retrieval for Super-Earths: Uniquely Constraining the Atmospheric Composition with Transmission Spectroscopy. 
ApJ753:100, DOI 10.1088/0004-637X/753/2/100, 1203.4018

23. Benz AO, Güdel M (1994) X-ray/microwave ratio of flares and coronae. A\&A285:621-630

24. Bergemann M, Ruchti G, Serenelli A, Feltzing S, Alvez-Brito A, Asplund M, Bensby T, Heiter U, Korn A, Lind K, Marino A, Jofre P, Nordlander T, Ryde N, Gilmore G, Randich S, Binney J, Bonifacio P, Drew J, Ferguson A, Jeffries R, Micela G, Negueruela I, Prusti T, Rix HW, Vallenari A, Alfaro Navarro E, Bragaglia A, Morbidelli L, Pancino E, Recio-Blanco A, Smiljanic R, Hill V, Lardo C, de Laverny P, Magrini L, Sacco G, Costado M, Kordopatis G, Tautvaisiene G (2014) The GaiaESO Survey: radial metallicity gradients and age-metallicity relation of stars in the Milky Way disk. ArXiv e-prints 1401.4437

25. Berger E (2002) Flaring up All Over-Radio Activity in Rapidly Rotating Late $M$ and L Dwarfs. ApJ572:503-513, DOI 10.1086/340301, astro-ph/0111317

26. Berger E (2006) Radio observations of a large sample of late $\mathrm{m}, 1$, and $\mathrm{t}$ dwarfs: The distribution of magnetic field strengths. ApJ 648:629-636, DOI 10.1086/505787, URL http://dx.doi.org/10.1086/505787

27. Berger E, Rutledge RE, Reid IN, Bildsten L, Gizis JE, Liebert J, Martín E, Basri G, Jayawardhana R, Brandeker A, Fleming TA, Johns-Krull CM, Giampapa MS, Hawley SL, Schmitt JHMM (2005) The Magnetic Properties of an L Dwarf Derived from Simultaneous Radio, X-Ray, and H $\alpha$ Observations. ApJ627:960-973, DOI 10.1086/430343, astro-ph/0502384

28. Berger E, Rutledge RE, Phan-Bao N, Basri G, Giampapa MS, Gizis JE, Liebert J, Martín E, Fleming TA (2009) Periodic Radio and $\mathrm{H} \alpha$ Emission from the L Dwarf Binary 2MASSW J0746425+200032: Exploring the Magnetic Field Topology and Radius Of An L Dwarf. ApJ695:310-316, DOI 10.1088/0004-637X/695/1/310, 0809.0001

29. Beuermann K, Dreizler S, Hessman FV, Backhaus U, Boesch A, Husser TO, Nortmann L, Schmelev A, Springer R (2013) The eclipsing post-common envelope binary CSS21055: a white dwarf with a probable brown-dwarf companion. A\&A558:A96, DOI 10.1051/0004-6361/201322241, 1312.5088

30. Bihain G, Rebolo R, Zapatero Osorio MR, Béjar VJS, Villó-Pérez I, Díaz-Sánchez A, Pérez-Garrido A, Caballero JA, Bailer-Jones CAL, Barrado y Navascués D, Eislöffel J, Forveille T, Goldman B, Henning T, Martín EL, Mundt R (2009) Candidate free-floating super-Jupiters in the young $\sigma$ Orionis open cluster. A\&A506:1169-1182, DOI 10.1051/0004-6361/200912210,0909.0802

31. Bilger C, Rimmer P, Helling C (2013) Small hydrocarbon molecules in cloudforming brown dwarf and giant gas planet atmospheres. MNRAS435:1888-1903, DOI 10.1093/mnras/stt1378, 1307.2565

32. Bonnefoy M, Marleau GD, Galicher R, Beust H, Lagrange AM, Baudino JM, Chauvin G, Borgniet S, Meunier N, Rameau J, Boccaletti A, Cumming A, Helling C, Homeier D, Allard F, Delorme P (2013) The near-infrared spectral energy distribution of $\beta$ Pictoris b. A\&A555:A107, DOI 10.1051/0004-6361/201220838, 1302.1160

33. Bozhinova I, Helling C, Scholz A (2014) The impact of M-dwarf atmosphere modelling on planet detection. MNRAS

34. Browning MK, Basri G, Marcy GW, West AA, Zhang J (2010) Rotation and 
Magnetic Activity in a Sample of M-Dwarfs. AJ139:504-518, DOI 10.1088/ 0004-6256/139/2/504

35. Buenzli E, Apai D, Morley CV, Flateau D, Showman AP, Burrows A, Marley MS, Lewis NK, Reid IN (2012) Vertical Atmospheric Structure in a Variable Brown Dwarf: Pressure-dependent Phase Shifts in Simultaneous Hubble Space TelescopeSpitzer Light Curves. ApJL760:L31, DOI 10.1088/2041-8205/760/2/L31, 1210. 6654

36. Buenzli E, Apai D, Radigan J, Reid IN, Flateau D (2014) Brown Dwarf Photospheres are Patchy: A Hubble Space Telescope Near-infrared Spectroscopic Survey Finds Frequent Low-level Variability. ApJ782:77, DOI 10.1088/0004-637X/782/2/77, 1312.5294

37. Burgasser AJ (2004) Discovery of a Second L Subdwarf in the Two Micron All Sky Survey. ApJL614:L73-L76, DOI 10.1086/425418, astro-ph/0409179

38. Burgasser AJ, Kirkpatrick JD (2006) Discovery of the Coolest Extreme Subdwarf. ApJ645:1485-1497, DOI 10.1086/504375, astro-ph/0603382

39. Burgasser AJ, Putman ME (2005) Quiescent Radio Emission from Southern LateType M Dwarfs and a Spectacular Radio Flare from the M8 Dwarf DENIS 10483956. ApJ626:486-497, DOI 10.1086/429788, astro-ph/0502365

40. Burgasser AJ, Kirkpatrick JD, Brown ME, Reid IN, Burrows A, Liebert J, Matthews K, Gizis JE, Dahn CC, Monet DG, Cutri RM, Skrutskie MF (2002) The Spectra of T Dwarfs. I. Near-Infrared Data and Spectral Classification. ApJ564:421-451, DOI 10.1086/324033, astro-ph/0108452

41. Burgasser AJ, Kirkpatrick JD, Burrows A, Liebert J, Reid IN, Gizis JE, McGovern MR, Prato L, McLean IS (2003) The First Substellar Subdwarf? Discovery of a Metal-poor L Dwarf with Halo Kinematics. ApJ592:1186-1192, DOI 10.1086/ 375813, astro-ph/0304174

42. Burgasser AJ, Geballe TR, Leggett SK, Kirkpatrick JD, Golimowski DA (2006) A Unified Near-Infrared Spectral Classification Scheme for T Dwarfs. ApJ637:10671093, DOI 10.1086/498563, astro-ph/0510090

43. Burgasser AJ, Vrba FJ, Lépine S, Munn JA, Luginbuhl CB, Henden AA, Guetter HH, Canzian BC (2008) Parallax and Luminosity Measurements of an L Subdwarf. ApJ672:1159-1166, DOI 10.1086/523810, 0709.1373

44. Burgasser AJ, Gillon M, Faherty JK, Radigan J, Amaury H M J, Plavchan P, Street R, Jehin E, Delrez L, Opitom C (2014) A Monitoring Campaign for Luhman 16AB. I. Detection of Resolved Near-Infrared Spectroscopic Variability. ArXiv e-prints 1402.2342

45. Burleigh MR, Hogan E, Dobbie PD, Napiwotzki R, Maxted PFL (2006) A nearinfrared spectroscopic detection of the brown dwarf in the post common envelope binary WD0137-349. MNRAS373:L55-L59, DOI 10.1111/j.1745-3933. 2006.00242.x, astro-ph/0609366

46. Burleigh MR, Steele PR, Dobbie PD, Farihi J, Napiwotzki R, Maxted PFL, Barstow MA, Jameson RF, Casewell SL, Gaensicke BT, Marsh TR (2011) Brown Dwarf Companions to White Dwarfs. In: Schuh S, Drechsel H, Heber U (eds) American Institute of Physics Conference Series, American Institute of Physics Conference Series, vol 1331, pp 262-270, DOI 10.1063/1.3556209, 1102.0505

47. Burningham B, Cardoso CV, Smith L, Leggett SK, Smart RL, Mann AW, Dhital 
S, Lucas PW, Tinney CG, Pinfield DJ, Zhang Z, Morley C, Saumon D, Aller K, Littlefair SP, Homeier D, Lodieu N, Deacon N, Marley MS, van Spaandonk L, Baker D, Allard F, Andrei AH, Canty J, Clarke J, Day-Jones AC, Dupuy T, Fortney JJ, Gomes J, Ishii M, Jones HRA, Liu M, Magazzú A, Marocco F, Murray DN, Rojas-Ayala B, Tamura M (2013) $76 \mathrm{~T}$ dwarfs from the UKIDSS LAS: benchmarks, kinematics and an updated space density. MNRAS433:457-497, DOI $10.1093 / \mathrm{mnras} / \mathrm{stt} 740,1304.7246$

48. Burningham B, Smith L, Cardoso CV, Lucas PW, Burgasser AJ, Jones HRA, Smart RL (2014) The discovery of a T6.5 subdwarf. ArXiv e-prints 1401.5982

49. Burrows A, Hubbard WB, Lunine JI (1989) Theoretical models of very low mass stars and brown dwarfs. ApJ345:939-958, DOI 10.1086/167964

50. Burrows A, Hubbard WB, Lunine JI, Liebert J (2001) The theory of brown dwarfs and extrasolar giant planets. Reviews of Modern Physics 73:719-765, DOI 10. 1103/RevModPhys.73.719, astro-ph/0103383

51. Burrows A, Burgasser AJ, Kirkpatrick JD, Liebert J, Milsom JA, Sudarsky D, Hubeny I (2002) Theoretical Spectral Models of T Dwarfs at Short Wavelengths and Their Comparison with Data. ApJ573:394-417, DOI 10.1086/340584, astro-ph/0109227

52. Burrows A, Sudarsky D, Lunine JI (2003) Beyond the T Dwarfs: Theoretical Spectra, Colors, and Detectability of the Coolest Brown Dwarfs. ApJ596:587-596, DOI $10.1086 / 377709$, astro-ph/0304226

53. Burrows A, Sudarsky D, Hubeny I (2006) L and T Dwarf Models and the L to T Transition. ApJ640:1063-1077, DOI 10.1086/500293, astro-ph/0509066

54. Burrows A, Heng K, Nampaisarn T (2011) The Dependence of Brown Dwarf Radii on Atmospheric Metallicity and Clouds: Theory and Comparison with Observations. ApJ736:47, DOI 10.1088/0004-637X/736/1/47, 1102.3922

55. Casewell SL, Dobbie PD, Hodgkin ST, Moraux E, Jameson RF, Hambly NC, Irwin J, Lodieu N (2007) Proper motion L and T dwarf candidate members of the Pleiades. MNRAS378:1131-1140, DOI 10.1111/j.1365-2966.2007.11848.x, 0704.1578

56. Casewell SL, Jameson RF, Burleigh MR, Dobbie PD, Roy M, Hodgkin ST, Moraux E (2011) Methane band and Spitzer mid-IR imaging of $\mathrm{L}$ and $\mathrm{T}$ dwarf candidates in the Pleiades. MNRAS412:2071-2078, DOI 10.1111/j.1365-2966.2010.18044. $\mathrm{x}, 1011.4218$

57. Casewell SL, Burleigh MR, Wynn GA, Alexander RD, Napiwotzki R, Lawrie KA, Dobbie PD, Jameson RF, Hodgkin ST (2012) WD0837+185: The Formation and Evolution of an Extreme Mass-ratio White-dwarf-Brown-dwarf Binary in Praesepe. ApJL759:L34, DOI 10.1088/2041-8205/759/2/L34, 1210.0446

58. Casewell SL, Burleigh MR, Lawrie KA, Maxted PFL, Dobbie PD, Napiwotzki R (2013) Irradiated brown dwarfs . Mem. Societa Astronomica Italiana84:1022, 1401.1405

59. Catlow CRA, Bromley ST, Hamad S, Mora-Fonz M, Sokol AA, Woodley SM (2010) Modelling nano-clusters and nucleation. Physical Chemistry Chemical Physics (Incorporating Faraday Transactions) 12:786, DOI 10.1039/b916069h

60. Chabrier G, Baraffe I, Allard F, Hauschildt P (2000) Evolutionary Models for Very Low-Mass Stars and Brown Dwarfs with Dusty Atmospheres. ApJ542:464-472, DOI 10.1086/309513, astro-ph/0005557 
61. Chabrier G, Johansen A, Janson M, Rafikov R (2014) Giant planet and brown dwarf formation. ArXiv e-prints 1401.7559

62. Cheng JY, Rockosi CM, Morrison HL, Schönrich RA, Lee YS, Beers TC, Bizyaev D, Pan K, Schneider DP (2012) Metallicity Gradients in the Milky Way Disk as Observed by the SEGUE Survey. ApJ746:149, DOI 10.1088/0004-637X/746/2/ $149,1110.5933$

63. Clarke FJ, Oppenheimer BR, Tinney CG (2002) A mini-survey for variability in early L dwarfs. MNRAS335:1158-1162, DOI 10.1046/j.1365-8711.2002.05691. $\mathrm{x}$, astro-ph/0205463

64. Clarke FJ, Hodgkin ST, Oppenheimer BR, Robertson J, Haubois X (2008) A search for J-band variability from late-L and T brown dwarfs. MNRAS386:2009-2014, DOI 10.1111/j.1365-2966.2008.13135.x

65. Cooper CS, Sudarsky D, Milsom JA, Lunine JI, Burrows A (2003) Modeling the Formation of Clouds in Brown Dwarf Atmospheres. ApJ586:1320-1337, DOI $10.1086 / 367763$

66. Crossfield IJM, Biller B, Schlieder JE, Deacon NR, Bonnefoy M, Homeier D, Allard F, Buenzli E, Henning T, Brandner W, Goldman B, Kopytova T (2014) A global cloud map of the nearest known brown dwarf. Nature505:654-656, DOI 10.1038/ nature12955, 1401.8145

67. Cruz KL, Kirkpatrick JD, Burgasser AJ (2009) Young L Dwarfs Identified in the Field: A Preliminary Low-Gravity, Optical Spectral Sequence from L0 to L5. AJ137:3345-3357, DOI 10.1088/0004-6256/137/2/3345, 0812.0364

68. Currie T, Burrows A, Itoh Y, Matsumura S, Fukagawa M, Apai D, Madhusudhan N, Hinz PM, Rodigas TJ, Kasper M, Pyo TS, Ogino S (2011) A Combined Subaru/VLT/MMT 1-5 $\mu \mathrm{m}$ Study of Planets Orbiting HR 8799: Implications for Atmospheric Properties, Masses, and Formation. ApJ729:128, DOI 10.1088/0004-637X/729/2/128, 1101.1973

69. Cushing MC, Looper D, Burgasser AJ, Kirkpatrick JD, Faherty J, Cruz KL, Sweet A, Sanderson RE (2009) 2MASS J06164006-6407194: The First Outer Halo L Subdwarf. ApJ696:986-993, DOI 10.1088/0004-637X/696/1/986, 0902.1059

70. Cushing MC, Saumon D, Marley MS (2010) SDSS J141624.08+134826.7: Blue L dwarfs and Non-equilibrium Chemistry. AJ140:1428-1432, DOI 10.1088/ 0004-6256/140/5/1428, 1009.2802

71. Cushing MC, Kirkpatrick JD, Gelino CR, Griffith RL, Skrutskie MF, Mainzer A, Marsh KA, Beichman CA, Burgasser AJ, Prato LA, Simcoe RA, Marley MS, Saumon D, Freedman RS, Eisenhardt PR, Wright EL (2011) The Discovery of Y Dwarfs using Data from the Wide-field Infrared Survey Explorer (WISE). ApJ743:50, DOI 10.1088/0004-637X/743/1/50, 1108.4678

72. Cushing MC, Kirkpatrick JD, Gelino CR, Mace GN, Skrutskie MF, Gould A (2014) Three New Cool Brown Dwarfs Discovered with the Wide-field Infrared Survey Explorer (WISE) and an Improved Spectrum of the Y0 Dwarf WISE J041022.71+150248.4. ArXiv e-prints 1402.1378

73. Delfosse X, Forveille T, Perrier C, Mayor M (1998) Rotation and chromospheric activity in field M dwarfs. A\&A331:581-595

74. Dieterich SB, Henry TJ, Jao WC, Winters JG, Hosey AD, Riedel AR, Subasavage JP (2013) The Solar Neighborhood XXXII. The Hydrogen Burning Limit. ArXiv 
e-prints 1312.1736

75. Dobbie PD, Burleigh MR, Levan AJ, Barstow MA, Napiwotzki R, Holberg JB, Hubeny I, Howell SB (2005) A near-infrared spectroscopic search for very-lowmass cool companions to notable DA white dwarfs. MNRAS357:1049-1058, DOI 10.1111/j.1365-2966.2005.08720.x, astro-ph/0412222

76. Dupuy TJ, Kraus AL (2013) Distances, Luminosities, and Temperatures of the Coldest Known Substellar Objects. Science 341:1492-1495, DOI 10.1126/science. 1241917, 1309.1422

77. Dupuy TJ, Liu MC, Bowler BP, Cushing MC, Helling C, Witte S, Hauschildt P (2010) Studying the Physical Diversity of Late-M Dwarfs with Dynamical Masses. ApJ721:1725-1747, DOI 10.1088/0004-637X/721/2/1725, 1007.4197

78. Enoch ML, Brown ME, Burgasser AJ (2003) Photometric Variability at the L/T Dwarf Boundary. AJ126:1006-1016, DOI 10.1086/376598, astro-ph/0305048

79. Faherty JK, Cruz KL, Rice EL, Riedel A (2013a) Young brown dwarfs as giant exoplanet analogs . Mem. Societa Astronomica Italiana84:955, 1307.8413

80. Faherty JK, Rice EL, Cruz KL, Mamajek EE, Núñez A (2013b) 2MASS J035523.37+113343.7: A Young, Dusty, Nearby, Isolated Brown Dwarf Resembling a Giant Exoplanet. AJ145:2, DOI 10.1088/0004-6256/145/1/2, 1206.5519

81. Faherty JK, Rice EL, Cruz KL, Mamajek EE, Núñez A (2013c) 2MASS J035523.37+113343.7: A Young, Dusty, Nearby, Isolated Brown Dwarf Resembling a Giant Exoplanet. AJ145:2, DOI 10.1088/0004-6256/145/1/2, 1206.5519

82. Farihi J, Christopher M (2004) A Possible Brown Dwarf Companion to the White Dwarf GD 1400. AJ128:1868-1871, DOI 10.1086/423919, astro-ph/0407036

83. Fletcher LN, Orton GS, Yanamandra-Fisher P, Fisher BM, Parrish PD, Irwin PGJ (2009) Retrievals of atmospheric variables on the gas giants from ground-based mid-infrared imaging. Icarus200:154-175, DOI 10.1016/j.icarus.2008.11.019

84. Fletcher LN, Baines KH, Momary TW, Showman AP, Irwin PGJ, Orton GS, RoosSerote M, Merlet C (2011) Saturn's tropospheric composition and clouds from Cassini/VIMS 4.6-5.1 $\mu \mathrm{m}$ nightside spectroscopy. Icarus214:510-533, DOI 10. 1016/j.icarus.2011.06.006

85. Fortney JJ, Lodders K, Marley MS, Freedman RS (2008) A Unified Theory for the Atmospheres of the Hot and Very Hot Jupiters: Two Classes of Irradiated Atmospheres. ApJ678:1419-1435, DOI 10.1086/528370,0710.2558

86. Freytag B, Allard F, Ludwig HG, Homeier D, Steffen M (2010) The role of convection, overshoot, and gravity waves for the transport of dust in $\mathrm{M}$ dwarf and brown dwarf atmospheres. A\&A513:A19, DOI 10.1051/0004-6361/200913354, 1002.3437

87. Geballe TR, Knapp GR, Leggett SK, Fan X, Golimowski DA, Anderson S, Brinkmann J, Csabai I, Gunn JE, Hawley SL, Hennessy G, Henry TJ, Hill GJ, Hindsley RB, Ivezić Ž, Lupton RH, McDaniel A, Munn JA, Narayanan VK, Peng E, Pier JR, Rockosi CM, Schneider DP, Smith JA, Strauss MA, Tsvetanov ZI, Uomoto A, York DG, Zheng W (2002) Toward Spectral Classification of L and T Dwarfs: Infrared and Optical Spectroscopy and Analysis. ApJ564:466-481, DOI 10.1086/324078, astro-ph/0108443

88. Gelino CR, Marley MS, Holtzman JA, Ackerman AS, Lodders K (2002) L Dwarf Variability: I-Band Observations. ApJ577:433-446, DOI 10.1086/342150, 
astro-ph/0205305

89. Gillon M, Triaud AHMJ, Jehin E, Delrez L, Opitom C, Magain P, Lendl M, Queloz D (2013) Fast-evolving weather for the coolest of our two new substellar neighbours. A\&A555:L5, DOI 10.1051/0004-6361/201321620, 1304.0481

90. Girardin F, Artigau É, Doyon R (2013) In Search of Dust Clouds: Photometric Monitoring of a Sample of Late L and T Dwarfs. ApJ767:61, DOI 10.1088/0004-637X/ $767 / 1 / 61$

91. Gizis JE (1997) M-Subdwarfs: Spectroscopic Classification and the Metallicity Scale. AJ113:806-822, DOI 10.1086/118302, astro-ph/9611222

92. Gizis JE, Monet DG, Reid IN, Kirkpatrick JD, Liebert J, Williams RJ (2000) New Neighbors from 2MASS: Activity and Kinematics at the Bottom of the Main Sequence. AJ120:1085-1099, DOI 10.1086/301456, astro-ph/0004361

93. Gizis JE, Shipman HL, Harvin JA (2005) First Ultraviolet Spectrum of a Brown Dwarf: Evidence for $\mathrm{H}_{2}$ Fluorescence and Accretion. ApJL630:L89-L91, DOI 10.1086/462414, astro-ph/0507429

94. Göres A (1993) The Formation of PAHs in C-Type Star Environments. In: Klare G (ed) Reviews in Modern Astronomy, Reviews in Modern Astronomy, vol 6, pp 165-178

95. Güdel M, Benz AO (1993) X-ray/microwave relation of different types of active stars. ApJL405:L63-L66, DOI 10.1086/186766

96. Gustafsson B, Edvardsson B, Eriksson K, Jørgensen UG, Nordlund Å, Plez B (2008) A grid of MARCS model atmospheres for late-type stars. I. Methods and general properties. A\&A486:951-970, DOI 10.1051/0004-6361:200809724, 0805.0554

97. Güttler C, Blum J, Zsom A, Ormel CW, Dullemond CP (2010) The outcome of protoplanetary dust growth: pebbles, boulders, or planetesimals?. I. Mapping the zoo of laboratory collision experiments. A\&A513:A56, DOI 10.1051/0004-6361/ 200912852,0910.4251

98. Hall PB (2002) 2MASSI J1315309-264951: An L Dwarf with Strong and Variable H $\alpha$ Emission. ApJL564:L89-L92, DOI 10.1086/339020, astro-ph/0112241

99. Hallinan G, Antonova A, Doyle JG, Bourke S, Brisken WF, Golden A (2006) Rotational modulation of the radio emission from the $\mathrm{m} 9$ dwarf tvlm 513-46546: Broadband coherent emission at the substellar boundary? ApJ 653:690-699, DOI 10.1086/508678, URL http://dx.doi.org/10.1086/508678

100. Hallinan G, Bourke S, Lane C, Antonova A, Zavala RT, Brisken WF, Boyle RP, Vrba FJ, Doyle JG, Golden A (2007) Periodic Bursts of Coherent Radio Emission from an Ultracool Dwarf. ApJL663:L25-L28, DOI 10.1086/519790, 0705. 2054

101. Hallinan G, Antonova A, Doyle JG, Bourke S, Lane C, Golden A (2008) Confirmation of the Electron Cyclotron Maser Instability as the Dominant Source of Radio Emission from Very Low Mass Stars and Brown Dwarfs. ApJ 684:644-653, DOI 10.1086/590360, URL http://adsabs.harvard.edu/abs/2008ApJ . . $684 \ldots 644 \mathrm{H}, 0805.4010$

102. Heinze AN, Metchev S, Apai D, Flateau D, Kurtev R, Marley M, Radigan J, Burgasser AJ, Artigau É, Plavchan P (2013) Weather on Other Worlds. I. Detection of Periodic Variability in the L3 Dwarf DENIS-P J1058.7-1548 with Precise Multi-wavelength Photometry. ApJ767:173, DOI 10.1088/0004-637X/767/2/173, 1303.2948 
103. Helling C, Fomins A (2013) Modelling the formation of atmospheric dust in brown dwarfs and planetary atmospheres. Royal Society of London Philosophical Transactions Series A 371:10,581, DOI 10.1098/rsta.2011.0581

104. Helling C, Woitke P (2006) Dust in brown dwarfs. V. Growth and evaporation of dirty dust grains. A\&A455:325-338, DOI 10.1051/0004-6361:20054598

105. Helling C, Oevermann M, Lüttke MJH, Klein R, Sedlmayr E (2001) Dust in brown dwarfs. I. Dust formation under turbulent conditions on microscopic scales. A\&A376:194-212, DOI 10.1051/0004-6361:20010937

106. Helling C, Klein R, Woitke P, Nowak U, Sedlmayr E (2004) Dust in brown dwarfs. IV. Dust formation and driven turbulence on mesoscopic scales. A\&A423:657675, DOI 10.1051/0004-6361:20034514, astro-ph/0404272

107. Helling C, Ackerman A, Allard F, Dehn M, Hauschildt P, Homeier D, Lodders K, Marley M, Rietmeijer F, Tsuji T, Woitke P (2008a) A comparison of chemistry and dust cloud formation in ultracool dwarf model atmospheres. MNRAS391:1854 1873, DOI 10.1111/j.1365-2966.2008.13991.x, 0809.3657

108. Helling C, Dehn M, Woitke P, Hauschildt PH (2008b) Consistent Simulations of Substellar Atmospheres and Nonequilibrium Dust Cloud Formation. ApJL675:L105-L108, DOI 10.1086/533462,0801.3733

109. Helling C, Woitke P, Thi WF (2008c) Dust in brown dwarfs and extra-solar planets. I. Chemical composition and spectral appearance of quasi-static cloud layers. A\&A485:547-560, DOI 10.1051/0004-6361:20078220, 0803.4315

110. Helling C, Jardine M, Mokler F (2011a) Ionization in Atmospheres of Brown Dwarfs and Extrasolar Planets. II. Dust-induced Collisional Ionization. ApJ737:38, DOI 10.1088/0004-637X/737/1/38, 1105.4409

111. Helling C, Jardine M, Witte S, Diver DA (2011b) Ionization in Atmospheres of Brown Dwarfs and Extrasolar Planets. I. The Role of Electron Avalanche. ApJ727:4, DOI 10.1088/0004-637X/727/1/4, 1010.4389

112. Helling C, Jardine M, Stark C, Diver D (2013) Ionization in Atmospheres of Brown Dwarfs and Extrasolar Planets. III. Breakdown Conditions for Mineral Clouds. ApJ767:136, DOI 10.1088/0004-637X/767/2/136, 1301.7586

113. Henry RBC, Worthey G (1999) The Distribution of Heavy Elements in Spiral and Elliptical Galaxies. PASP111:919-945, DOI 10.1086/316403, astro-ph/ 9904017

114. Hill C, Yurchenko SN, Tennyson J (2013) Temperature-dependent molecular absorption cross sections for exoplanets and other atmospheres. Icarus226:16731677, DOI 10.1016/j.icarus.2012.07.028, 1205.6514

115. Iraci LT, Phebus BD, Stone BM, Colaprete A (2010) Water ice cloud formation on Mars is more difficult than presumed: Laboratory studies of ice nucleation on surrogate materials. Icarus210:985-991, DOI 10.1016/j.icarus.2010.07.020

116. James DJ, Jardine MM, Jeffries RD, Randich S, Collier Cameron A, Ferreira M (2000) X-ray emission from nearby M-dwarfs: the super-saturation phenomenon. MNRAS318:1217-1226, DOI 10.1046/j.1365-8711.2000.03838.x, astro-ph/ 0007159

117. Joergens V (ed) (2014) 50 Years of Brown Dwarfs: From Prediction to Discovery to Forefront of Research, Astrophysics and Space Science Library, vol 401, DOI 10.1007/978-3-319-01162-2 
118. Khandrika H, Burgasser AJ, Melis C, Luk C, Bowsher E, Swift B (2013) A Search for Photometric Variability in L- and T-type Brown Dwarf Atmospheres. AJ145:71, DOI 10.1088/0004-6256/145/3/71, 1301.0545

119. Kirkpatrick JD (2013) Cold brown dwarfs with WISE: Y dwarfs and the field mass function. Astronomische Nachrichten 334:26-31, DOI 10.1002/asna.201211759

120. Kirkpatrick JD, Reid IN, Liebert J, Cutri RM, Nelson B, Beichman CA, Dahn CC, Monet DG, Gizis JE, Skrutskie MF (1999) Dwarfs Cooler than "M": The Definition of Spectral Type "L" Using Discoveries from the 2 Micron All-Sky Survey (2MASS). ApJ519:802-833, DOI 10.1086/307414

121. Kirkpatrick JD, Cruz KL, Barman TS, Burgasser AJ, Looper DL, Tinney CG, Gelino CR, Lowrance PJ, Liebert J, Carpenter JM, Hillenbrand LA, Stauffer JR (2008) A Sample of Very Young Field L Dwarfs and Implications for the Brown Dwarf "Lithium Test" at Early Ages. ApJ689:1295-1326, DOI 10.1086/592768, 0808.3153

122. Kirkpatrick JD, Looper DL, Burgasser AJ, Schurr SD, Cutri RM, Cushing MC, Cruz KL, Sweet AC, Knapp GR, Barman TS, Bochanski JJ, Roellig TL, McLean IS, McGovern MR, Rice EL (2010) Discoveries from a Near-infrared Proper Motion Survey Using Multi-epoch Two Micron All-Sky Survey Data. ApJS190:100 146, DOI 10.1088/0067-0049/190/1/100, 1008.3591

123. Kirkpatrick JD, Cushing MC, Gelino CR, Griffith RL, Skrutskie MF, Marsh KA, Wright EL, Mainzer A, Eisenhardt PR, McLean IS, Thompson MA, Bauer JM, Benford DJ, Bridge CR, Lake SE, Petty SM, Stanford SA, Tsai CW, Bailey V, Beichman CA, Bloom JS, Bochanski JJ, Burgasser AJ, Capak PL, Cruz KL, Hinz PM, Kartaltepe JS, Knox RP, Manohar S, Masters D, Morales-Calderón M, Prato LA, Rodigas TJ, Salvato M, Schurr SD, Scoville NZ, Simcoe RA, Stapelfeldt KR, Stern D, Stock ND, Vacca WD (2011) The First Hundred Brown Dwarfs Discovered by the Wide-field Infrared Survey Explorer (WISE). ApJS197:19, DOI 10.1088/0067-0049/197/2/19, 1108.4677

124. Kirkpatrick JD, Gelino CR, Cushing MC, Mace GN, Griffith RL, Skrutskie MF, Marsh KA, Wright EL, Eisenhardt PR, McLean IS, Mainzer AK, Burgasser AJ, Tinney CG, Parker S, Salter G (2012) Further Defining Spectral Type "Y" and Exploring the Low-mass End of the Field Brown Dwarf Mass Function. ApJ753:156, DOI 10.1088/0004-637X/753/2/156, 1205.2122

125. Kirkpatrick JD, Cushing MC, Gelino CR, Beichman CA, Tinney CG, Faherty JK, Schneider A, Mace GN (2013) Discovery of the Y1 Dwarf WISE J064723.23623235.5. ApJ776:128, DOI 10.1088/0004-637X/776/2/128, 1308.5372

126. Kirkpatrick JD, Schneider A, Fajardo-Acosta S, Gelino CR, Mace GN, Wright EL, Logsdon SE, McLean IS, Cushing MC, Skrutskie MF, Eisenhardt PR, Stern D, Balokovic M, Burgasser AJ, Faherty JK, Lansbury GB, Rich JA, Skrzypek N, Fowler JW, Cutri RM, Masci FJ, Conrow T, Grillmair CJ, McCallon HL, Beichman CA, Marsh KA (2014) The AllWISE Motion Survey and The Quest for Cold Subdwarfs. ArXiv e-prints 1402.0661

127. Knutson HA, Charbonneau D, Cowan NB, Fortney JJ, Showman AP, Agol E, Henry GW, Everett ME, Allen LE (2009) Multiwavelength Constraints on the Day-Night Circulation Patterns of HD 189733b. ApJ690:822-836, DOI 10.1088/0004-637X/ 690/1/822,0802.1705 
128. Konopka U, Mokler F, Ivlev AV, Kretschmer M, Morfill GE, Thomas HM, Rothermel H, Fortov VE, Lipaev AM, Molotkov VI, Nefedov AP, Baturin YM, Budarin Y, Ivanov AI, Roth M (2005) Charge-induced gelation of microparticles. New Journal of Physics 7:227, DOI 10.1088/1367-2630/7/1/227

129. Lee JM, Heng K, Irwin PGJ (2013) Atmospheric Retrieval Analysis of the Directly Imaged Exoplanet HR 8799b. ApJ778:97, DOI 10.1088/0004-637X/778/2/97, 1307.1404

130. Lépine S, Rich RM, Shara MM (2007) Revised Metallicity Classes for Low-Mass Stars: Dwarfs (dM), Subdwarfs (sdM), Extreme Subdwarfs (esdM), and Ultrasubdwarfs (usdM). ApJ669:1235-1247, DOI 10.1086/521614,0707.2993

131. Liebert J, Kirkpatrick JD, Cruz KL, Reid IN, Burgasser A, Tinney CG, Gizis JE (2003) A Flaring L5 Dwarf: The Nature of $\mathrm{H} \alpha$ Emission in Very Low Mass (Sub)Stellar Objects. AJ125:343-347, DOI 10.1086/345514, astro-ph/0210348

132. Line MR, Fortney JJ, Marley MS, Sorahana S (2014) An Approach for Retrieving Temperatures and Abundances in Brown Dwarf Atmospheres. ArXiv e-prints 1403.6412

133. Littlefair SP, Dhillon VS, Marsh TR, Shahbaz T, Martín EL, Copperwheat C (2008) Optical variability of the ultracool dwarf TVLM 513-46546: evidence for inhomogeneous dust clouds. MNRAS391:L88-L92, DOI 10.1111/j.1745-3933.2008. 00562.x, 0809.2193

134. Liu MC, Dupuy TJ, Leggett SK (2010) Discovery of a Highly Unequal-mass Binary T Dwarf with Keck Laser Guide Star Adaptive Optics: A Coevality Test of Substellar Theoretical Models and Effective Temperatures. ApJ722:311-328, DOI 10.1088/0004-637X/722/1/311, 1008.2200

135. Lodders K, Fegley B (2002) Atmospheric Chemistry in Giant Planets, Brown Dwarfs, and Low-Mass Dwarf Stars. I. Carbon, Nitrogen, and Oxygen. Icarus155:393-424, DOI 10.1006/icar.2001.6740

136. Lodders K, Fegley B Jr (2006) Chemistry of Low Mass Substellar Objects, p 1. DOI 10.1007/3-540-30313-8_1

137. Lodieu N, Zapatero Osorio MR, Martín EL, Solano E, Aberasturi M (2010) GTC/OSIRIS Spectroscopic Identification of a Faint L Subdwarf in the UKIRT Infrared Deep Sky Survey. ApJL708:L107-L111, DOI 10.1088/2041-8205/708/2/ L107, 0912.3364

138. Lodieu N, Espinoza Contreras M, Zapatero Osorio MR, Solano E, Aberasturi M, Martín EL (2012) New ultracool subdwarfs identified in large-scale surveys using Virtual Observatory tools. I. UKIDSS LAS DR5 vs. SDSS DR7. A\&A542:A105, DOI 10.1051/0004-6361/201118717, 1204.4328

139. Lucas PW, Roche PF, Allard F, Hauschildt PH (2001) Infrared spectroscopy of substellar objects in Orion. MNRAS326:695-721, DOI 10.1046/j.1365-8711.2001. 04666.x, astro-ph/0105154

140. Ludwig HG, Allard F, Hauschildt PH (2002) Numerical simulations of surface convection in a late M-dwarf. A\&A395:99-115, DOI 10.1051/0004-6361:20021153, astro-ph/0208584

141. Luhman KL (2012) The Formation and Early Evolution of Low-Mass Stars and Brown Dwarfs. ARA\&A50:65-106, DOI 10.1146/annurev-astro-081811-125528, 1208.5800 
142. Luhman KL (2013) Discovery of a Binary Brown Dwarf at 2 pe from the Sun. ApJL767:L1, DOI 10.1088/2041-8205/767/1/L1, 1303.2401

143. Luhman KL (2014) Discovery of a $250 \mathrm{~K}$ Brown Dwarf at 2 pc from the Sun. ApJL786:L18, DOI 10.1088/2041-8205/786/2/L18, 1404.6501

144. Luhman KL, Burgasser AJ, Bochanski JJ (2011) Discovery of a Candidate for the Coolest Known Brown Dwarf. ApJL730:L9, DOI 10.1088/2041-8205/730/1/L9, 1102.5411

145. Luhman KL, Burgasser AJ, Labbé I, Saumon D, Marley MS, Bochanski JJ, Monson AJ, Persson SE (2012) Confirmation of One of the Coldest Known Brown Dwarfs. ApJ744:135, DOI 10.1088/0004-637X/744/2/135, 1110.4353

146. Lunine JI, Hubbard WB, Marley MS (1986) Evolution and infrared spectra of brown dwarfs. ApJ310:238-260, DOI 10.1086/164678

147. Mace GN, Kirkpatrick JD, Cushing MC, Gelino CR, McLean IS, Logsdon SE, Wright EL, Skrutskie MF, Beichman CA, Eisenhardt PR, Kulas KR (2013) The Exemplar T8 Subdwarf Companion of Wolf 1130. ApJ777:36, DOI 10.1088/ 0004-637X/777/1/36, 1309.1500

148. Madhusudhan N, Seager S (2009) A Temperature and Abundance Retrieval Method for Exoplanet Atmospheres. ApJ707:24-39, DOI 10.1088/0004-637X/707/1/24, 0910.1347

149. Madhusudhan N, Burrows A, Currie T (2011) Model Atmospheres for Massive Gas Giants with Thick Clouds: Application to the HR 8799 Planets and Predictions for Future Detections. ApJ737:34, DOI 10.1088/0004-637X/737/1/34, 1102.5089

150. Marley MS, Saumon D, Goldblatt C (2010) A Patchy Cloud Model for the L to T Dwarf Transition. ApJL723:L117-L121, DOI 10.1088/2041-8205/723/1/L117, 1009.6217

151. Marley MS, Saumon D, Cushing M, Ackerman AS, Fortney JJ, Freedman R (2012) Masses, Radii, and Cloud Properties of the HR 8799 Planets. ApJ754:135, DOI 10.1088/0004-637X/754/2/135, 1205.6488

152. Marley MS, Ackerman AS, Cuzzi JN, Kitzmann D (2013) Clouds and Hazes in Exoplanet Atmospheres. ArXiv e-prints 1301.5627

153. Martín EL, Delfosse X, Basri G, Goldman B, Forveille T, Zapatero Osorio MR (1999) Spectroscopic Classification of Late-M and L Field Dwarfs. AJ118:24662482, DOI 10.1086/301107

154. Maxted PFL, Napiwotzki R, Dobbie PD, Burleigh MR (2006) Survival of a brown dwarf after engulfment by a red giant star. Nature442:543-545, DOI 10.1038/nature04987, astro-ph/0608054

155. McLean M, Berger E, Irwin J, Forbrich J, Reiners A (2011) Periodic Radio Emission from the M7 Dwarf 2MASS J13142039+1320011: Implications for the Magnetic Field Topology. ApJ741:27, DOI 10.1088/0004-637X/741/1/27, 1107.1516

156. Metchev S, Apai D, Radigan J, Artigau É, Heinze A, Helling C, Homeier D, Littlefair S, Morley C, Skemer A, Stark C (2013) Clouds in brown dwarfs and giant planets. Astronomische Nachrichten 334:40-43, DOI 10.1002/asna.201211776

157. Mihalas DM (1982) Stellar atmospheres.

158. Mohanty S, Basri G, Shu F, Allard F, Chabrier G (2002) Activity in Very Cool Stars: Magnetic Dissipation in Late M and L Dwarf Atmospheres. ApJ571:469486, DOI 10.1086/339911, astro-ph/0201518 
159. Morin J, Donati JF, Petit P, Delfosse X, Forveille T, Jardine MM (2010) Large-scale magnetic topologies of late M dwarfs. MNRAS407:2269-2286, DOI 10.1111/j. 1365-2966.2010.17101.x, 1005.5552

160. Morioka A, Miyoshi Y, Kitamura N, Misawa H, Tsuchiya F, Menietti JD, Honary F (2012) Fundamental characteristics of field-aligned auroral acceleration derived from akr spectra. JGR 117(A2):A02,213, DOI 10.1029/2011ja017137, URL http://dx.doi.org/10.1029/2011ja017137

161. Morley CV, Fortney JJ, Marley MS, Visscher C, Saumon D, Leggett SK (2012) Neglected Clouds in T and Y Dwarf Atmospheres. ApJ756:172, DOI 10.1088/ 0004-637X/756/2/172, 1206.4313

162. Morley CV, Marley MS, Fortney JJ, Lupu R (2014a) Spectral Variability from the Patchy Atmospheres of T and Y Dwarfs. ApJL1406.0863

163. Morley CV, Marley MS, Fortney JJ, Lupu R, Saumon D, Greene T, Lodders K (2014b) Water Clouds in Y Dwarfs and Exoplanets. ApJ 1404.0005

164. Moses JI, Visscher C, Fortney JJ, Showman AP, Lewis NK, Griffith CA, Klippenstein SJ, Shabram M, Friedson AJ, Marley MS, Freedman RS (2011) Disequilibrium Carbon, Oxygen, and Nitrogen Chemistry in the Atmospheres of HD 189733b and HD 209458b. ApJ737:15, DOI 10.1088/0004-637X/737/1/15, 1102.0063

165. Nakajima T, Oppenheimer BR, Kulkarni SR, Golimowski DA, Matthews K, Durrance ST (1995) Discovery of a cool brown dwarf. Nature378:463-465, DOI 10.1038/378463a0

166. Nichols JD, Burleigh MR, Casewell SL, Cowley SWH, Wynn GA, Clarke JT, West AA (2012) Origin of electron cyclotron maser induced radio emissions at ultracool dwarfs: Magnetosphere-ionosphere coupling currents. ApJ 760(1):59, DOI 10. 1088/0004-637x/760/1/59, URL http://dx.doi.org/10.1088/0004-637x/ $760 / 1 / 59$

167. Noll KS, Geballe TR, Marley MS (1997) Detection of Abundant Carbon Monoxide in the Brown Dwarf Gliese 229B. ApJL489:L87, DOI 10.1086/310954

168. Noyes RW, Hartmann LW, Baliunas SL, Duncan DK, Vaughan AH (1984) Rotation, convection, and magnetic activity in lower main-sequence stars. ApJ279:763-777, DOI 10.1086/161945

169. Osten RA, Hawley SL, Bastian TS, Reid IN (2006) The Radio Spectrum of TVLM 513-46546: Constraints on the Coronal Properties of a Late M Dwarf. ApJ637:518-521, DOI 10.1086/498345, astro-ph/0509762

170. Patience J, King RR, De Rosa RJ, Vigan A, Witte S, Rice E, Helling C, Hauschildt P (2012) Spectroscopy across the brown dwarf/planetary mass boundary. I. Nearinfrared JHK spectra. A\&A540:A85, DOI 10.1051/0004-6361/201118058, 1201. 3921

171. Peña Ramírez K, Béjar VJS, Zapatero Osorio MR, Petr-Gotzens MG, Martín EL (2012) New Isolated Planetary-mass Objects and the Stellar and Substellar Mass Function of the $\sigma$ Orionis Cluster. ApJ754:30, DOI 10.1088/0004-637X/754/1/30, 1205.4950

172. Pereira TMD, Asplund M, Collet R, Thaler I, Trampedach R, Leenaarts J (2013) How realistic are solar model atmospheres? A\&A554:A118, DOI 10.1051/ 0004-6361/201321227, 1304.4932 
173. Pinfield DJ, Jones HRA, Lucas PW, Kendall TR, Folkes SL, Day-Jones AC, Chappelle RJ, Steele IA (2006) Finding benchmark brown dwarfs to probe the substellar initial mass function as a function of time. MNRAS368:1281-1295, DOI 10.1111/j.1365-2966.2006.10213.x, astro-ph/0603320

174. Pizzolato N, Maggio A, Micela G, Sciortino S, Ventura P (2003) The stellar activity-rotation relationship revisited: Dependence of saturated and non-saturated X-ray emission regimes on stellar mass for late-type dwarfs. A\&A397:147-157, DOI 10.1051/0004-6361:20021560

175. Radigan J, Jayawardhana R, Lafrenière D, Artigau É, Marley M, Saumon D (2012) Large-amplitude Variations of an L/T Transition Brown Dwarf: Multi-wavelength Observations of Patchy, High-contrast Cloud Features. ApJ750:105, DOI 10.1088/ 0004-637X/750/2/105, 1201.3403

176. Radigan J, Lafrenière D, Jayawardhana R, Artigau E (2014) Strong Brightness Variations Signal Cloudy-to-Clear Transition of Brown Dwarfs. ArXiv e-prints 1404.3247

177. Rajpurohit AS, Reylé C, Schultheis M, Leinert C, Allard F, Homeier D, Ratzka T, Abraham P, Moster B, Witte S, Ryde N (2012) The very low mass multiple system LHS 1070. A testbed for model atmospheres for the lower end of the main sequence. A\&A545:A85, DOI 10.1051/0004-6361/201219029, 1208.0452

178. Ravi V, Hallinan G, Hobbs G, Champion DJ (2011) The Magnetosphere of the Ultracool Dwarf DENIS 1048-3956. ApJL735:L2, DOI 10.1088/2041-8205/735/ 1/L2, 1105.0990

179. Rebolo R, Zapatero Osorio MR, Martín EL (1995) Discovery of a brown dwarf in the Pleiades star cluster. Nature377:129-131, DOI 10.1038/377129a0

180. Reid IN, Burgasser AJ, Cruz KL, Kirkpatrick JD, Gizis JE (2001) Near-Infrared Spectral Classification of Late M and L Dwarfs. AJ121:1710-1721, DOI 10.1086/ 319418, astro-ph/0012275

181. Reid IN, Cruz KL, Kirkpatrick JD, Allen PR, Mungall F, Liebert J, Lowrance P, Sweet A (2008) Meeting the Cool Neighbors. X. Ultracool Dwarfs from the 2MASS All-Sky Data Release. AJ136:1290-1311, DOI 10.1088/0004-6256/136/ 3/1290

182. Reiners A, Basri G, Christensen UR (2009) Surprisingly Weak Magnetism on Young Accreting Brown Dwarfs. ApJ697:373-379, DOI 10.1088/0004-637X/697/ $1 / 373,0903.0857$

183. Rice EL, Faherty JK, Cruz K, Barman T, Looper D, Malo L, Mamajek EE, Metchev S, Shkolnik EL (2011) Juvenile Ultracool Dwarfs. In: Johns-Krull C, Browning MK, West AA (eds) 16th Cambridge Workshop on Cool Stars, Stellar Systems, and the Sun, Astronomical Society of the Pacific Conference Series, vol 448, p 481, 1101.4231

184. Rimmer PB, Helling C (2013) Ionization in Atmospheres of Brown Dwarfs and Extrasolar Planets. IV. The Effect of Cosmic Rays. ApJ774:108, DOI 10.1088/ 0004-637X/774/2/108, 1307.3257

185. Rimmer PB, Helling C, Bilger C (2014) The Influence of Galactic Cosmic Rays on Ion-Neutral Hydrocarbon Chemistry in the Upper Atmospheres of Free-Floating Exoplanets. ArXiv e-prints 1312.1138

186. Robinson TD, Marley MS (2014) Temperature Fluctuations as a Source of Brown 
Dwarf Variability. ArXiv e-prints 1403.2438

187. Rojas-Ayala B, Hilton EJ, Mann AW, Lépine S, Gaidos E, Bonfils X, Helling C, Henry TJ, Rogers LA, von Braun K, Youdin A (2013) M dwarf stars in the light of (future) exoplanet searches. Astronomische Nachrichten 334:155, DOI 10.1002/ asna.201211760, 1211.0544

188. Rossow WB (1978) Cloud microphysics - Analysis of the clouds of Earth, Venus, Mars, and Jupiter. Icarus36:1-50, DOI 10.1016/0019-1035(78)90072-6

189. Route M, Wolszczan A (2012) The arecibo detection of the coolest radio-flaring brown dwarf. ApJL 747(2):L22, DOI 10.1088/2041-8205/747/2/122, URL http: //dx.doi.org/10.1088/2041-8205/747/2/122

190. Sarro LM, Berihuete A, Carrión C, Barrado D, Cruz P, Isasi Y (2013) Properties of ultra-cool dwarfs with Gaia. An assessment of the accuracy for the temperature determination. A\&A550:A44, DOI 10.1051/0004-6361/201219867, 1212.3096

191. Saumon D, Bergeron P, Lunine JI, Hubbard WB, Burrows A (1994) Cool zerometallicity stellar atmospheres. ApJ424:333-344, DOI 10.1086/173892

192. Saumon D, Geballe TR, Leggett SK, Marley MS, Freedman RS, Lodders K, Fegley B Jr, Sengupta SK (2000) Molecular Abundances in the Atmosphere of the T Dwarf GL 229B. ApJ541:374-389, DOI 10.1086/309410, astro-ph/0003353

193. Saunders RW, Forster PM, Plane JMC (2007) Potential climatic effects of meteoric smoke in the Earth's paleo-atmosphere. Geophysical Review Letter 34:L16801, DOI 10.1029/2007GL029648

194. Schmidt SJ, Cruz KL, Bongiorno BJ, Liebert J, Reid IN (2007) Activity and Kinematics of Ultracool Dwarfs, Including an Amazing Flare Observation. AJ133:2258-2273, DOI 10.1086/512158, astro-ph/0701055

195. Showman AP, Kaspi Y (2013a) Atmospheric Dynamics of Brown Dwarfs and Directly Imaged Giant Planets. ApJ776:85, DOI 10.1088/0004-637X/776/2/85, 1210.7573

196. Showman AP, Kaspi Y (2013b) Atmospheric Dynamics of Brown Dwarfs and Directly Imaged Giant Planets. ApJ776:85, DOI 10.1088/0004-637X/776/2/85, 1210.7573

197. Sinclair JA, Helling C, Greaves JS (2010) The impact of stellar model spectra in disc detection. MNRAS409:L49-L53, DOI 10.1111/j.1745-3933.2010.00945.x

198. Sivarani T, Lépine S, Kembhavi AK, Gupchup J (2009) SDSS J125637022452: A High Proper Motion L Subdwarf. ApJL694:L140-L143, DOI 10.1088/ 0004-637X/694/2/L140,0901.3382

199. Siverd RJ, et al (2012) KELT-1b: A Strongly Irradiated, Highly Inflated, Short Period, 27 Jupiter-mass Companion Transiting a Mid-F Star. ApJ761:123, DOI 10.1088/0004-637X/761/2/123, 1206.1635

200. Sorahana S, Yamamura I, Murakami H (2013) On the Radii of Brown Dwarfs Measured with AKARI Near-infrared Spectroscopy. ApJ767:77, DOI 10.1088/ 0004-637X/767/1/77, 1304.1259

201. Sorahana S, Suzuki TK, Yamamura I (2014) A Signature of Chromospheric Activity in Brown Dwarfs Revealed by 2.5-5.0 Micron AKARI Spectra. ArXiv e-prints 1401.5801

202. Southworth J (2009) Homogeneous studies of transiting extrasolar planets II. Physical properties. MNRAS394:272-294, DOI 10.1111/j.1365-2966.2008. 
14274.x, 0811.3277

203. Spezzi L, Alves de Oliveira C, Moraux E, Bouvier J, Winston E, Hudelot P, Bouy H, Cuillandre JC (2012) Searching for planetary-mass T-dwarfs in the core of Serpens. A\&A545:A105, DOI 10.1051/0004-6361/201219559, 1208.0702

204. Stark CR, Helling C, Diver DA, Rimmer PB (2013) Ionization in Atmospheres of Brown Dwarfs and Extrasolar Planets. V. Alfvén Ionization. ApJ776:11, DOI 10.1088/0004-637X/776/1/11, 1308.2991

205. Steele PR, Saglia RP, Burleigh MR, Marsh TR, Gänsicke BT, Lawrie K, Cappetta M, Girven J, Napiwotzki R (2013) NLTT 5306: the shortest period detached white dwarf+brown dwarf binary. MNRAS429:3492-3500, DOI 10.1093/mnras/sts620, 1212.2899

206. Stelzer B, Neuhäuser R (2003) X-ray Emission from Old and Intermediate Age Brown Dwarfs. In: Martín E (ed) Brown Dwarfs, IAU Symposium, vol 211, p 443, astro-ph/0206284

207. Stelzer B, Micela G, Flaccomio E, Neuhäuser R, Jayawardhana R (2006) X-ray emission of brown dwarfs: towards constraining the dependence on age, luminosity, and temperature. A\&A448:293-304, DOI 10.1051/0004-6361:20053677, astro-ph/0511168

208. Stewart RT, Innis JL, Slee OB, Nelson GJ, Wright AE (1988) A relation between radio luminosity and rotation for late-type stars. AJ96:371-377, DOI $10.1086 / 114815$

209. Tinney CG, Tolley AJ (1999a) Searching for weather in brown dwarfs. MNRAS304:119-126, DOI 10.1046/j.1365-8711.1999.02297.x, astro-ph/9809165

210. Tinney CG, Tolley AJ (1999b) Searching for weather in brown dwarfs. MNRAS304:119-126, DOI 10.1046/j.1365-8711.1999.02297.x, astro-ph/9809165

211. Treumann R (2006) The electron-cyclotron maser for astrophysical application. A\&A Rev 13(4):229-315, DOI 10.1007/s00159-006-0001-y, URL http://dx. doi.org/10.1007/s00159-006-0001-y

212. Triaud AHMJ, Gillon M, Selsis F, Winn JN, Demory BO, Artigau E, Laughlin GP, Seager S, Helling C, Mayor M, Albert L, Anderson RI, Bolmont E, Doyon R, Forveille T, Hagelberg J, Leconte J, Lendl M, Littlefair S, Raymond S, Sahlmann J (2013) A search for rocky planets transiting brown dwarfs. ArXiv e-prints 1304 . 7248

213. Tsuji T (2001) Unified Model Photospheres for Ultracool Dwarfs of Types L and T. In: Jones HRA, Steele IA (eds) Ultracool Dwarfs: New Spectral Types L and T, p 9, astro-ph/0103395

214. Tsuji T (2002) Dust in the Photospheric Environment: Unified Cloudy Models of M, L, and T Dwarfs. ApJ575:264-290, DOI 10.1086/341262, astro-ph/ 0204401

215. Tsuji T (2005) Dust in the Photospheric Environment. III. A Fundamental Element in the Characterization of Ultracool Dwarfs. ApJ621:1033-1048, DOI 10.1086/ 427747, astro-ph/0411766

216. Tsuji T, Ohnaka K, Aoki W (1996a) Dust formation in stellar photospheres: a case of very low mass stars and a possible resolution on the effective temperature scale 
of M dwarfs. A\&A305:L1

217. Tsuji T, Ohnaka K, Aoki W, Nakajima T (1996b) Evolution of dusty photospheres through red to brown dwarfs: how dust forms in very low mass objects. A\&A308:L29-L32

218. Tsuji T, Nakajima T, Yanagisawa K (2004) Dust in the Photospheric Environment. II. Effect on the Near-Infrared Spectra of L and T Dwarfs. ApJ607:511-529, DOI 10.1086/383300, astro-ph/0402192

219. Tsuji T, Yamamura I, Sorahana S (2011) AKARI Observations of Brown Dwarfs. II. $\mathrm{CO}_{2}$ as Probe of Carbon and Oxygen Abundances in Brown Dwarfs. ApJ734:73, DOI 10.1088/0004-637X/734/2/73, 1105.6303

220. Venot O, Hébrard E, Agúndez M, Dobrijevic M, Selsis F, Hersant F, Iro N, Bounaceur R (2012) A chemical model for the atmosphere of hot Jupiters. A\&A546:A43, DOI 10.1051/0004-6361/201219310, 1208.0560

221. Wada K, Tanaka H, Okuzumi S, Kobayashi H, Suyama T, Kimura H, Yamamoto $\mathrm{T}$ (2013) Growth efficiency of dust aggregates through collisions with high mass ratios. A\&A559:A62, DOI 10.1051/0004-6361/201322259

222. Williams PKG (2013) The observed rotation/activity relations of ultracool dwarfs. Mem. Societa Astronomica Italiana84:1122

223. Williams PKG, Berger E, Zauderer BA (2013a) Quasi-quiescent Radio Emission from the First Radio-emitting T Dwarf. ApJL767:L30, DOI 10.1088/2041-8205/ 767/2/L30, 1301.2321

224. Williams PKG, Cook BA, Berger E (2013b) Trends in Ultracool Dwarf Magnetism. I. X-Ray Suppression and Radio Enhancement. ArXiv e-prints 1310.6757

225. Wilson PA, Rajan A, Patience J (2014) The brown dwarf atmosphere monitoring (BAM) project. I. The largest near-IR monitoring survey of $\mathrm{L}$ and $\mathrm{T}$ dwarfs. A\&A566:A111, DOI 10.1051/0004-6361/201322995, 1404.4633

226. Witte S, Helling C, Hauschildt PH (2009) Dust in brown dwarfs and extrasolar planets. II. Cloud formation for cosmologically evolving abundances. A\&A506:1367-1380, DOI 10.1051/0004-6361/200811501,0908.3597

227. Witte S, Helling C, Barman T, Heidrich N, Hauschildt PH (2011) Dust in brown dwarfs and extra-solar planets. III. Testing synthetic spectra on observations. A\&A529:A44, DOI 10.1051/0004-6361/201014105

228. Woitke $\mathrm{P}$ (2006) Too little radiation pressure on dust in the winds of oxygen-rich AGB stars. A\&A460:L9-L12, DOI 10.1051/0004-6361:20066322, astro-ph/ 0609392

229. Woitke P, Helling C (2003) Dust in brown dwarfs. II. The coupled problem of dust formation and sedimentation. A\&A399:297-313, DOI 10.1051/0004-6361: 20021734

230. Woitke P, Helling C (2004) Dust in brown dwarfs. III. Formation and structure of quasi-static cloud layers. A\&A414:335-350, DOI 10.1051/0004-6361:20031605

231. Yamamura I, Tsuji T, Tanabé T (2010) AKARI Observations of Brown Dwarfs. I. $\mathrm{CO}$ and $\mathrm{CO}_{2}$ Bands in the Near-infrared Spectra. ApJ722:682-698, DOI 10.1088/ 0004-637X/722/1/682, 1008.3732

232. Yuan TT, Kewley LJ, Swinbank AM, Richard J, Livermore RC (2011) Metallicity Gradient of a Lensed Face-on Spiral Galaxy at Redshift 1.49. ApJL732:L14, DOI 10.1088/2041-8205/732/1/L14, 1103.3277 
233. Zhang X, Showman AP (2014) Atmospheric Circulation of Brown Dwarfs: Jets, Vortices, and Time Variability. ArXiv e-prints 1403.2143 